\title{
The Assortment Packing Problem: Multiperiod Assortment Planning for Short-Lived Products
}

\author{
Felipe Caro*
}

Victor Martínez-de-Albéniz ${ }^{\dagger}$

April 26, 2014
Paat Rusmevichientong $\ddagger$

\begin{abstract}
Motivated by retailers' frequent introduction of new items to refresh product lines and maintain their market shares, we present the assortment packing problem in which a firm must decide, in advance, the release date of each product in a given collection over a selling season. Our formulation models the trade-offs among profit margins, preference weights, and limited life cycles. A key aspect of the problem is that each product is short-lived in the sense that, once introduced, its attractiveness lasts only a few periods and vanishes over time. The objective is to determine when to introduce each product to maximize the total profit over the selling season. Even for two periods, the corresponding optimization problem is shown to be NP-complete. As a result, we study a continuous relaxation of the problem that approximates the problem well, when the number of products is large. When margins are identical and product preferences decay exponentially, its solution can be characterized: it is optimal to introduce products with slower decays earlier. The structural properties of the relaxation also help us to develop several heuristics, for which we establish performance guarantees. We test our heuristics with data on sales and release dates of woman handbags from an accessories retailer. The numerical experiments show that the heuristics perform very well and can yield significant improvements in profitability.
\end{abstract}

\section{Introduction}

Keeping customers interested is one of the challenges in industries with short-lived products. Firms launch products frequently in order to keep their presence in the market place and capture the attention of customers. This forces firms to plan their assortments over time. Indeed, carrying a static assortment - one that remains the same over time - becomes ineffective and possibly unprofitable because consumers are quickly "bored" with the choices within assortment and they divert their purchases to other consumption options. In other words, the customers' preference for a particular product in the assortment decays over time, as it ages on the shelf. Moreover, due to substitution effects, it might not be optimal for a firm to release all the products at once because cannibalization may dilute its market share, so timing product entry becomes relevant.

An industry where assortment renewal strategies have been massively adopted is apparel retailing. The industry traditionally used to launch two collections a year and push them to the stores at the beginning of the Spring and Autumn (specifically, once clearance sales conclude). Over the last decade, powerful players such as the Swedish clothing retailer H\&M has chosen to continuously release their products into the stores. $\mathrm{H} \& \mathrm{M}$ claims that it introduces new products into stores

\footnotetext{
*UCLA Anderson School of Management, Los Angeles, CA 90095, fcaro@anderson.ucla.edu

${ }^{\dagger}$ IESE Business School, University of Navarra, Barcelona, Spain, valbeniz@iese.edu

${ }^{\ddagger}$ USC Marshall School of Business, Los Angeles, CA 90089, rusmevic@marshall.usc.edu
} 
daily (H\&M 2007). Interestingly, the timing of release is not necessarily linked to when a product is designed or produced. Indeed, H\&M is well known for selling mostly basic and fashion-basic items, which are products with low or medium fashion content respectively, e.g., neutral colors such as black and white, standard cuts and few embellishments such as ribbons (see Abernathy et al. 1999 for a more detailed discussion on basics and fashion-basics). These products are typically designed and ordered long in advance, but are only required at the store in the middle of the selling season. Similarly, Florida-based Chico's claims that "You'll find something new every day at Chico's", and the new products introduced in store have a six-month lead time, which means that the company decouples design decisions and introduction timing (Tiplady 2006).

Besides apparel, other industries exhibit similar dynamics. Book stores typically announce and promote "recent arrivals" but then move them to less prominent locations because the attractiveness of the product quickly drops after introduction. Restaurants also frequently change the items on their menu to avoid customer satiation, with some restaurants committing to completely change the menu every three months (Moskin 2011). Finally, motion picture distributors schedule the release dates of their movies over a season. Since customers and theaters tend to prefer new releases, it is important to avoid having too many movies in the theaters at the same time to prevent cannibalization of ticket sales (The Economist 2007).

While this trend seems to provide better values to consumers from a marketing standpoint, it leads to a more complex execution from an operations perspective because the firm needs to decide how to deploy a catalog of products in stores over a season. Traditionally, the entire catalog was pushed to the store in the first day of the season, and would not be changed over time. However, such a strategy is no longer appropriate when products are short-lived. Indeed, under this strategy, the store would have a big spike in sales at the beginning of the season but then it would see that its market share drastically shrinks. In contrast, a strategy of continuously releasing a few products every period would keep some products' valuation high, balancing the low valuation of items that were introduced long ago, maintaining the firm's market share later in the season.

With a large number of products, determining each product's release date in order to maximize the total profit over a season is challenging. In practice, companies typically solve this problem manually, with a qualitative assessment of the value of the assortment for each week. For example, we have interacted with a Spanish accessories retailer that introduced a large number of items at the beginning of the season and then added a few items to the assortment each week. The decision process was completely manual, and it was based purely on price and visual characteristics of the items. How to arrange product introductions over time constitutes a difficult multiperiod 
assortment planning problem. Our objective in this paper is to shed some light on the issue, and we (i) develop a model to capture the trade-offs related to product introductions, (ii) provide exact or approximate solutions to support introduction decisions, and (iii) derive some managerial insights that highlight the importance of incorporating preference decay when timing product releases.

For this purpose, we present the assortment packing problem in which a planner must decide, in advance, when to introduce each product in a given collection over a selling season. The products have different profit margins, preference weights, and life cycle patterns. Once a product is introduced to the store, the product becomes stale and its attractiveness decays over time. The objective is to determine if and when to release each product to maximize the total profit over the selling season. We model demand in the form of market shares following an attraction model (Bell et al. 1975). The model takes as its inputs each product's profit margin, its initial preference weight (i.e., the value of the product in the attraction model when it is introduced), and its preference decay (i.e., the decay of the product's preference weight over time). As an output, the model determines the the release date of each product over the season that maximizes the firm's discounted profit.

When the season has a single period, the assortment packing problem reduces to a classical static assortment optimization problem under the attraction demand model, which can be solved efficiently (see, for example, Talluri and van Ryzin 2004, Kök et al. 2008). However, even with just two periods, the problem becomes NP-hard and the particular structure found in the one-period assortment problem is lost. As a result, we study a relaxation of the problem that corresponds to the situation where there are many different products of each type, and we show that it approximates the original problem well. We can characterize the optimal solution of this relaxation and find that, when margins are identical and product preferences decay exponentially, it is optimal to introduce products sequentially, in a decreasing order of preference decay, i.e., always introduce a product with slowest decay first. We then build several heuristics to solve the original problem, for which a performance guarantee can be derived. As shown in our numerical experiments, these heuristics perform remarkably well and can yield significant improvements in profitability.

Our work thus contributes to the literature in three dimensions: modeling, methodological development, and managerial insights. While there has been a number of papers on understanding the benefits of frequent assortment changes on demand learning (see, for example, Caro and Gallien 2007, Alptekinoglu et al. 2012), there has been less attention on multiperiod assortment planning when learning is not a predominant issue. In that context, we provide a formulation that explicitly models when each product is introduced, and determines the optimal assortment in each period taking into account the natural decay that occurs over time. 


\section{Literature Review}

This paper is closely related to the literature on assortment planning and product rollover in operations management. Our work extends the static assortment planning problem to a multiperiod setting. Kök et al. (2008) provide an excellent review of the literature in this area. The assortment optimization problem is typically driven by the trade-off between product margins and sales. Indeed, a larger assortment induces a higher sales probability, but at the same time, it also includes lower-margin items. Talluri and van Ryzin (2004) first show in a single-period revenue management setting that the optimal assortment is a set comprising of highest-margin products. In more general contexts, the assortment problem does not have a simple solution. For instance, Bront et al. (2009) show that when there is an heterogenous population the single-period problem becomes NP-Hard.

An important stream of operations papers focus on the relationship between variety benefits and inventory costs, and they typically assume a certain product substitution structure when stockouts occur (see, for example, van Ryzin and Mahajan 1999, Smith and Agrawal 2000). In most of these papers, the inventory decision follows the assortment decision, in the sense that a newsvendor-type formula is used to compute the inventory cost for a given assortment. As a result, the evolution of the assortment (as some products "die") is ignored. There are some exceptions: Mahajan and van Ryzin (2001) show that when substitution is stockout-based, the problem quickly becomes intractable and profits are no longer concave in inventory levels; Honhon et al. (2010) propose a dynamic programming formulation to find the optimal stocking levels when there is a fixed proportion of customer types and random demand; Bernstein et al. (2010) dynamically adjust the assortment over time, depending on each customer's preferences and the remaining inventory, so as to "hide" products with low inventories to reserve them for future customers. Furthermore, pricing decisions have also been considered: Aydin and Porteus (2008) study the joint inventory and pricing decision for an assortment. The competitive aspect of these assortment and price decisions has been studied in Besbes and Sauré (2010), under the multinomial logit (MNL) model. In our paper, we consider the assortment decision only, in a multiperiod environment.

Dynamic assortments have been studied before, usually in a context where the underlying demand function is unknown and must be estimated, and inventory is ignored. Caro and Gallien (2007) apply a finite-horizon multi-armed bandit model with Bayesian learning to a dynamic assortment problem for seasonal goods, and derive a closed-form dynamic index policy that captures the key exploration versus exploitation tradeoffs. Rusmevichientong et al. (2010) and Sauré and Zeevi (2008) consider the case of MNL demand model with a capacity constraint, and present an assortment planning algorithm that simultaneously learns the underlying MNL parameters and 
optimizes profit. Farias and Madan (2011) consider the setting where a product cannot be used once it is removed from the assortment. Farias et al. (2010) consider the problem of estimating a choice model that is defined as a distribution over all permutations, where each permutation defines a preference-ordered list of products. Alptekinoglu et al. (2012) also learn about customer preferences in a locational model by dynamically adjusting the assortment. Caro and Martínezde-Albéniz (2012) analyze how satiation effects influence customer decisions over time, and lead retailers to offer fresher products under competition. Honhon and Kök (2011) study the impact of variety-seeking customers and identify cyclical patterns in the optimal assortment. All of the papers mentioned thus far assume that the attractiveness of each product remains the same over time. To our knowledge, we are the first to explicitly model the reduction of a product's attractiveness over its life cycle: we propose an attraction model in which product preference weights decay over time. A similar approach has been used in Ainslie et al. (2005), where box-office sales are modeled as a MNL demand model where the market attractiveness of each movie follows a Gamma distribution of its age in the theaters (and hence eventually decays over time). That paper provides the empirical methodology that can potentially be used to calibrate the decay parameters in our assortment packing model. It does not study how to time movie releases, which is the focus of our work.

In addition, product introduction timing has also been studied by the product rollover literature. Most of the work relevant to this paper studies the trade-off between the market expansion associated with a new product and the saturation effect, i.e., how the older product is cannibalized by the new one. Lim and Tang (2006) study whether introducing a new product and removing an older one should be done simultaneously or not, and characterize the best product prices associated with these strategies. Demand diffusion models have also been used to analyze the timing decision. For instance, Kalish et al. (1995) study whether two markets need to be entered simultaneously or sequentially. With a similar diffusion pattern, Savin and Terwiesch (2005) study the timing of entry for a new product competing against an established one. Druehl et al. (2009) analyze the pace of product introduction over multiple product generations with market growth, cannibalization, margin decays and pace-dependent introduction costs.

\section{The Assortment Packing Problem}

In this section, we describe the assortment packing problem and discuss the features of our model in detail. Note that all the proofs are contained in the Appendix.

3.1 Model Formulation. Consider a retailer that is planning the assortment for a season consisting of $T$ periods (e.g., weeks). The retailer has $n$ products available, and must decide when to introduce each product $i \in I:=\{1, \ldots, n\}$. For $i \in I$ and $t=1, \ldots, T$, let $x_{i t}$ be the binary 
variable that is equal to 1 if product $i$ is introduced in period $t$, and 0 otherwise. Each product can only be introduced once; after it is introduced, it remains in the assortment for several consecutive periods. Let $r_{i}$ denote the unit gross margin of product $i$. Without loss of generality, assume that the products are numbered so that such that $r_{1} \geq r_{2} \geq \ldots \geq r_{n}>0$. Period $t$ has a discount or seasonality factor $\alpha_{t}$.

We assume an attraction demand model, where each product's market share contribution is proportional to its preference weight or attractiveness in each period. Let $v_{i}$ denote the weight of product $i$ when it is first introduced. As the product remains in the store, however, its attractiveness changes over time. For instance, the product might become stale, in which case its attractiveness decays as time goes by. If product $i$ is introduced in period $t_{i}$, then we assume that its weight in period $t \geq t_{i}$ is given by $\kappa_{i, t-t_{i}} v_{i}$, where $\kappa_{i, d} \in[0,1]$ is a product-specific decay parameter. ${ }^{1}$ In particular, if the item has a planned life cycle of $\ell_{i}$ periods, then $\kappa_{i, d}=0$ for $d \geq \ell_{i}$. In any period, a customer can choose not to purchase from the assortment. This outside option has a weight $v_{0}$, independent of time. As a result, the assortment packing problem $(A P P)$ can be formulated as the following nonlinear combinatorial optimization problem:

$$
\begin{aligned}
& V^{*}=\max \sum_{t=1}^{T} \alpha_{t} \sum_{i=1}^{n} r_{i} \times\left(\frac{v_{i} \sum_{u=1}^{t} \kappa_{i, t-u} x_{i u}}{v_{0}+\sum_{j=1}^{n} v_{j} \sum_{u=1}^{t} \kappa_{j, t-u} x_{j u}}\right) \\
& \text { s.t. } \quad \sum_{t=1}^{T} x_{i t} \leq 1 \quad \forall i \in I \text {, } \\
& x_{i t} \in\{0,1\}
\end{aligned}
$$

The objective function (1) is the sum of the discounted (gross) profits over all products from each of the $T$ periods in the season. The expression in the parentheses represents the market share of product $i$ in period $t$. Constraint (2) ensures that each product is introduced at most once. Note that one could impose $x_{i 1}=\ldots=x_{i t-1}=0$, for some $t>1$ and let the summation in constraint (2) start in period $t$ if there is a product that is in the production pipeline and will become available later in the season. Finally, constraint (3) imposes the binary requirement on the decision variables.

For notational convenience, let $z_{i t}:=\sum_{u=1}^{t} \kappa_{i, t-u} x_{i u}$ denote the contribution of product $i$ to the assortment's attractiveness or load in period $t$. Clearly, $z_{i t}=0$ if product $i$ is introduced after period $t$. On the same lines, we define $z_{t}:=\sum_{i=1}^{n} v_{i} z_{i t}$ as the total load in period $t$ and $\phi_{t}:=z_{t} /\left(v_{0}+z_{t}\right)$ as the firm's market share in period $t$. Throughout the paper, we say that two products $i$ and $j$ have the same type if they have exactly the same decay pattern, i.e., $\kappa_{i, d}=\kappa_{j, d}$, for $d \geq 0$. In particular, we refer to products with no decay as basics. In other words, a product $i$

\footnotetext{
${ }^{1}$ We refer to $\kappa_{i, d}$ as a decay parameter though the model allows for general life cycle patterns.
} 
belongs to the basic type if $\kappa_{i, d}=1$ for all $d \geq 0$. This represents products with an attractiveness that might be low but remains constant from the release date until the end of the season, which is characteristic of basic apparel items as pointed out in the introduction. Interestingly, it is easy to show that when a basic product $i$ is such that $r_{i} \geq r_{j}$ for all $j$, then it is optimal to introduce it in the first period, and hence this product can be omitted from the analysis (although $i$ 's preference weight will still appear in the fractional terms of the objective functions).

3.2 Model Discussion. We review here the model's features, justify our modeling assumptions, and describe settings where our model might be applicable. We believe that this model strikes a good balance between maintaining tractability and capturing important constraints faced by the retailers in planning their product introductions.

First, we can see that the problem formulation requires that an open-loop policy is used. In other words, one must decide in advance when to introduce the different products, and cannot adjust release dates during the selling season. This assumption is motivated by applications where products have a long production lead time and a relatively short selling season, making it impractical to reorder products during the season. As pointed out in the introduction, many basic and fashion-basic apparel companies design the complete catalog of products before deciding the time of introduction. Furthermore, this is also a reasonable assumption for books, accessories or movies. As a result, since there is no possibility of adjusting the assortment for demand learning (in contrast to Caro and Gallien 2007), any uncertain demand can be incorporated into its deterministic counterpart, i.e., its expectation. ${ }^{2}$

One key characteristic of our model is the formulation of the demand function. We use an attraction model where the sales of product $j$ in time $t$ is given by $\alpha_{t} \times \frac{v_{i} z_{i t}}{v_{0}+\sum_{j=1}^{n} v_{j} z_{j t}}$. Such form is one of the most commonly used demand models in marketing and operations management to capture assortment-based substitution (Kök et al. 2008). In period $t$, a number $\alpha_{t}$ of consumers are ready to buy a product, and then choose product $i$ with probability $\frac{v_{i} z_{i t}}{v_{0}+\sum_{j=1}^{n} v_{j} z_{j t}}$. There are well-established techniques for estimating the initial attractiveness $v_{i}$ associated with each product (see e.g., Talluri and van Ryzin 2004). In addition, Ainslie et al. (2005) discuss the empirical toolbox for estimating the decay parameters $\kappa_{i, d}$, with applications to movie release dates. It is worth pointing out that Ainslie et al. (2005) assume that the weight of blockbuster movies decays

\footnotetext{
${ }^{2}$ We note that although the production decision might be decided in advance, in practice, this does not exclude the possibility of learning demand from alternative market signals, such as when products' attractiveness are correlated (so after a particular product is introduced, we can refine our estimates other products' preference weights), or when there is access to information on other markets (Elberse and Eliashberg 2003). However, in this paper, we focus on the problem where product introduction is being decided before the acquisition of new information, so we do not allow the retailer to modify the release schedule.
} 
exponentially, a functional form of $\kappa_{i, d}$ which we study in depth in Section 4.2.

In single-period models, $z_{i t}$ is either one if the product is introduced, or zero if it is not. In contrast, in our model, the contribution of each product depends on when the product was introduced. Specifically, if the product has not been introduced yet at time $t$, then $z_{i t}=0$; if the product has just been introduced at $t$, then $z_{i t}=1$; otherwise, the product has been in the assortment for a few periods, since $t_{i}$, and $z_{i t}=\kappa_{i, t-t_{i}} \leq 1$, which implies that the value of the item in the consumers' eyes is lower than at introduction. This feature captures the customers' preference for new items, which is prevalent for apparel, books, restaurant menus, or movie consumptions. As a result, it is clearly sub-optimal to introduce all the items in the first period. Indeed, if that was the chosen solution, sales would be high in the beginning of the horizon, but limited due to strong product competition; in contrast, they would be low at the end of the horizon, since the load of later periods $z_{t}$ would be much reduced compared to the outside option.

Furthermore, note that each product is introduced only once; it stays in the assortment until the end of the horizon. Limited product life cycles can be modeled by setting $\kappa_{i, d}=0$ for $d \geq \ell_{i}$ where $\ell_{i}$ is the planned life of the item. This implies that the product introduction decision is irrevocable, similar to Farias and Madan (2011). Although sometimes it may be possible to show a product to the customers, then remove it from the assortment and then reintroduce it again, this is usually not the case in retail settings: it is usually too complex and expensive to execute, involving handling, logistics and merchandizing costs. For instance, most apparel retailers introduce a product only once, and keep it in the stores for a number of periods. However, in Section 7 we consider a model extension that allows for product removals.

Finally, to keep the problem tractable and focused on the product introduction question, we do not consider inventory decisions. In practice, this is a decision that is taken after assortment plans are finalized, and hence can be set sufficiently high later on so as to avoid losing sales. Similarly, we do not consider budget or shelf-space constraints, e.g., a maximum number of products to be introduced over the horizon or a maximum number of items per period, and we assume a homogeneous population. These last two assumptions can be relaxed but we postpone that discussion until Section 7.

3.3 Computational Complexity. When there is no outside option $\left(v_{0}=0\right)$ and $\alpha_{t}=1$ for all $t$, the $A P P$ is trivial because it is optimal to introduce the most profitable product $r_{1}$ in the first period and to have it as the sole product in the assortment for the entire duration of its life cycle. The reason is that having an assortment with more products yields a per-period profit that is a weighted average of the individual margins $r_{1}, \ldots, r_{n}$, which clearly cannot exceed $r_{1}$. Once 
the attractiveness of the first product declines to zero, it is optimal to introduce the second most profitable product $r_{2}$, and so on and so forth.

Another case that can be solved efficiently is when all products are basic, with $\kappa_{i, d}=1$ for all $i$ and $d$. In this case, the optimal solution is myopic, and the problem is equivalent to a singleperiod setting with $T=1$. Talluri and van Ryzin (2004) show that for a single-period setting it is optimal to introduce products with the highest margins, corresponding to a revenue-ordered subset $A_{\ell}=\{1, \ldots, \ell\}$ for some product $\ell$. As shown in the following example, the nice structure is not present in the $A P P$ with decay parameters, and in general, it may be beneficial to introduce products with lower margins in the beginning of the season.

Example 1 (Lower Margin Products May Be Introduced First). Consider a setting with 2 products $(n=2)$ and 2 periods $(T=2)$, with no discounting $\left(\alpha_{1}=\alpha_{2}=1\right)$. Suppose that $r_{1}=\$ 10, r_{2}=\$ 9, v_{0}=1, v_{1}=3, v_{2}=7$, and $\kappa_{1, d}=\kappa_{2, d}=0.4^{d}$ for $d=0,1$. For this instance it can be verified that the optimal release schedule is to first introduce product 2 with lower margin in period 1 and only release the higher margin product (product 1) in period 2. Intuitively, by releasing product 1 later, it preserves its attractiveness, which allows for a higher profit from offering both products together.

The example above suggests that the general $A P P$ is computationally intractable, which is formally established below: Theorem 1 shows that, even when there is only two periods, no discount, all products have equal margin, and a single-period life cycle with $\kappa_{i, 0}=1$ and $\kappa_{i, d}=0$ for all $i$ and $d \geq 1$, the problem remains NP-hard. The proof follows from a reduction from the well known NP-complete PARTITIOn problem. We first formulate a decision-theoretic version of our two-period $A P P$.

Two-Period Equal-Margin Assortment Packing with One-Period Life Cycle

INPUTS: The set of products indexed by $i$; the preference weights $v_{1}, v_{2}, \ldots, v_{n}$, where $v_{i} \in \mathbb{Z}_{+}$for $i=1, \ldots, n$; a no-purchase weight $v_{0} \in \mathbb{Z}_{+}$; and the target profit $K \in \mathbb{Q}_{+}$;

Question: Is there a partition $S_{1}$ and $S_{2}$ such that $S_{1} \cap S_{2}=\varnothing$ and $S_{1} \cup S_{2}=\{1, \ldots, n\}$ such that

$$
\frac{\sum_{j \in S_{1}} v_{j}}{v_{0}+\sum_{j \in S_{1}} v_{j}}+\frac{\sum_{j \in S_{2}} v_{j}}{v_{0}+\sum_{j \in S_{2}} v_{j}} \geq K .
$$

Theorem 1. For any $v_{0} \in \mathbb{Z}_{+}$, the Two-Period Equal-Margin Assortment Packing with One-Period Life CyCle is NP-complete.

\section{The Continuous Relaxation}

In the previous section, we observe that the $A P P$ is computationally intractable even in the simplest setting, making it difficult to establish managerial insights and structural properties of the optimal 
solution, and to develop an algorithm for solving this problem. In this section, we introduce a continuous relaxation of the original problem, by replacing the binary constraints $x_{i t} \in\{0,1\}$ by $x_{i t} \geq 0$ for all $i$ and $t$. The corresponding continuous optimization problem is given by:

$$
\bar{V}=\max \left\{\sum_{t=1}^{T} \alpha_{t} \sum_{i=1}^{n} r_{i} \times\left(\frac{v_{i} \sum_{u=1}^{t} \kappa_{i, t-u} x_{i u}}{v_{0}+\sum_{j=1}^{n} v_{j} \sum_{u=1}^{t} \kappa_{j, t-u} x_{j u}}\right) \mid \sum_{t=1}^{T} x_{i t} \leq 1 \forall i, \quad x_{i t} \geq 0 \forall i, t\right\},
$$

and let $\left\{\bar{x}_{i t}: i=1, \ldots, n, t=1, \ldots, T\right\}$ be an optimal solution to the above problem.

When all products have equal margins, the above objective function is concave because the market share $\left(\sum_{i=1}^{n} v_{i} \sum_{u=1}^{t} \kappa_{i, t-u} x_{i u}\right) /\left(v_{0}+\sum_{j=1}^{n} v_{j} \sum_{u=1}^{t} \kappa_{j, t-u} x_{j u}\right)=\sum_{i=1}^{n} v_{i} z_{i t} /\left(v_{0}+\sum_{j=1}^{n} v_{j} z_{j t}\right)$ in each period $t$ is a concave function, and therefore the maximization can be carried out efficiently. When products have unequal margins, however, the continuous relaxation becomes a particular case of fractional programming known as the linear sum-of-ratios problem, see Schaible and Shi (2003), for which there is still no general polynomial optimization method but several branch-and-bound (b\&b) schemes have been developed, e.g., Kuno (2002) and Benson (2007).

Note that the continuous relaxation provides an upper bound $\bar{V}$ for the optimal value $V^{*}$ of the original $A P P$. However, it should be noted that there are instances where the objective value $\bar{V}$ is far from $V^{*}$. This happens when there is a "fat" item that is significantly more attractive that the rest, i.e., its weight $v_{i}$ is significantly larger than the weight of the other items (see Section 6 for some examples).

Another interesting feature of the relaxation problem is that all products of the same type with the same margin can be bundled together. Thus, if $r_{i}=r_{j}$ and $\kappa_{i, d}=\kappa_{j, d}$ for all $d \geq 0$, then we can combine products $i$ and $j$ and replace it with a new product having weight $v_{i}+v_{j}$, reducing the number of variables in the relaxation problem by one. Thus, the continuous relaxation can be seen as a strategic version of the original $A P P$ because it deals only with product types. The original $A P P$ is more of a tactical planning model, which might be used to determine when to release each specific product. The bundling of products with the same type and margin suggests that when we have a lot of products of each type, the continuous relaxation provides a good approximation to the original $A P P$, as shown in the following section.

4.1 A Fluid Approximation. The continuous relaxation has a fundamental interpretation as a fluid approximation of the $A P P$. To see this, consider a sequence of $A P P$ instances indexed by $k$, which we call $A P P_{k}, k=1,2, \ldots, \infty$, in which each original product $i$ is subdivided into $k$ subproducts with equal attractiveness $v_{i} / k$. Let $V_{k}$ be the optimal value of instance $k$. 


$$
\begin{aligned}
\left(A P P_{k}\right) \quad V_{k}=\max & \sum_{t=1}^{T} \alpha_{t} \sum_{i=1}^{n} r_{i} \times\left(\frac{\frac{v_{i}}{k} \sum_{u=1}^{t} \kappa_{i, t-u} \sum_{q=1}^{k} \nu_{i u q}}{v_{0}+\sum_{j=1}^{n} \frac{v_{j}}{k} \sum_{u=1}^{t} \kappa_{j, t-u} \sum_{q=1}^{k} \nu_{j u q}}\right) \\
\text { s.t. } & \sum_{t=1}^{T} \nu_{i t q} \leq 1 \quad \forall i \in I, q=1, \ldots, k, \\
& \nu_{i t q} \in\{0,1\} \quad \forall i \in I, t=1, \ldots, T, q=1, \ldots, k .
\end{aligned}
$$

All instances in the sequence $A P P_{k}$ have the same continuous relaxation, so $V_{k} \leq \bar{V}$ for all $k$. As the (sub)products become infinitesimally small, i.e, as they become a fluid, the objective value $V_{k}$ gets arbitrarily close to $\bar{V}$. The following theorem established the convergence rate to $\bar{V}$.

Theorem 2 (Fluid Approximation). For any $k \geq 1$,

$$
0 \leq \bar{V}-V_{k} \leq\left(\frac{\max _{j=1, \ldots, n} v_{j}}{k v_{0}+\max _{j=1, \ldots, n} v_{j}}\right) \bar{V},
$$

which implies that $\lim _{k \rightarrow \infty} V_{k} / \bar{V}=1$.

Theorem 2 tells us that when there are a lot of products of the same type with similar decay profiles, then we can obtain a good approximation to the $A P P$ by solving the continuous relaxation problem. Given the fluid interpretation, we proceed to analyze the continuous relaxation as a way to shed some light on the structure of the $A P P$, which we later use as guideline to develop general heuristics.

4.2 Exponential Preference Decay. We can provide structural results when the preference decay parameters are given by $\kappa_{i, d}=\kappa_{i}^{d}$ with $0<\kappa_{i} \leq 1$ and for all $i$ and $d \geq 0$. As discussed in Section 3.2, this is one of the most prevalent decay patterns observed in practice. In what follows, we first assume that all products have equal margins with $r_{i}=1$ for all $i$. Towards the end we discuss an extension with unequal margins.

Recall that for any product $i$ and time period $t, z_{i t}=\sum_{u=1}^{t} \kappa_{i, t-u} x_{i u}$ denote the load of product $i$ in period $t$. For any $t$, let $D_{t}=\frac{\alpha_{t} v_{0}}{\left(v_{0}+z_{t}\right)^{2}}$. Note that there is a one-to-one correspondence between $z_{t}=\sum_{i=1}^{n} v_{i} z_{i t}$ and $D_{t}$. We can roughly interpret $D_{t}$ as the marginal contribution to the total profit in period $t$. To see this, if $J:[0,1]^{n \times T} \rightarrow \mathbb{R}_{+}$denotes the objective function of the continuous 
relaxation problem, then

$$
J_{i, t}:=\frac{\partial J}{\partial x_{i t}}=v_{i} \sum_{l=t}^{T} \kappa_{i}^{l-t} D_{l}=v_{i} D_{t}+\kappa_{i} J_{i, t+1} .
$$

Since the continuous relaxation with equal margins is a concave optimization problem, we can use the Karush-Kuhn-Tucker (KKT) conditions with the dual variable $\lambda_{i} \geq 0$ associated with the constraint $\sum_{t=1}^{T} x_{i t} \leq 1$. At optimality, we then have the following system of equations:

$$
\lambda_{i} \geq J_{i, t} \quad i=1, \ldots, n, \quad t=1, \ldots, T, \quad \text { and } \quad 0=\lambda_{i}\left(1-\sum_{t=1}^{T} x_{i t}\right) \quad i=1, \ldots, n,
$$

along with the complementary slackness constraints that $x_{i t}\left(\lambda_{i}-J_{i, t}\right)=0$ for all $i$ and $t$. This implies that $\bar{x}_{i t}>0$ only if $J_{i, t}=v_{i} \sum_{l=t}^{T} \kappa_{i}^{l-t} D_{l}=\lambda_{i}$, and $\bar{x}_{i t}=0$ if $v_{i} \sum_{l=t}^{T} \kappa_{i}^{l-t} D_{l}<\lambda_{i}$. In other words, at optimality

$$
\frac{\lambda_{i}}{v_{i}}=\max _{t \geq 1}\left\{\sum_{l=t}^{T} \kappa_{i}^{l-t} D_{l}\right\}, \quad i=1, \ldots, n .
$$

As shown in Theorem 3 here below, by studying the structure of the dual variables, we can determine which products will be introduced first. Before stating the theorem, we will provide some intuition underlying our argument. Consider the simplest case with just two products where $\kappa_{1}>\kappa_{2}$, e.g., type 1 are regular staple items while type 2 are fashion-basic items such that their attractiveness is expected to fade away faster. To simplify our discussion, let us assume that $v_{1}=v_{2}=1$. Suppose that both products are introduced, we wish to show that the regular staple item (product 1) is introduced before the fashion-basic item (product 2).

Since $\kappa_{1}>\kappa_{2}$, it follows from Equation (5) that $\lambda_{1}>\lambda_{2}$. As shown in the proof of Theorem 3 in the Appendix, since both products are introduced, it must be the case that $\lambda_{1}\left(1-\kappa_{1}\right)<\lambda_{2}\left(1-\kappa_{2}\right)$. Moreover, from the KKT condition above, if a product $i$ is introduced in period $t$, with $x_{i t}>0$, then it must be the case that $D_{t-1} \leq \lambda_{i}\left(1-\kappa_{i}\right) \leq D_{t}$. Since $\lambda_{1}\left(1-\kappa_{1}\right)<\lambda_{2}\left(1-\kappa_{2}\right)$, product 1 must be introduced earlier (at least no later) than product 2, which is the desired result. In fact, this structure can be generalized to $n$ types of products: products are introduced in decreasing order of $\kappa_{i}$ over time, as shown in the following theorem.

Theorem 3 (Staggered Product Introduction). With equal margins, if $\kappa_{1}>\ldots>\kappa_{n}$ and $\alpha_{t}=\alpha^{t-1}$ for all $t$ with $\alpha \leq 1$, then for each product $i \in I$ there exists a time window $\left[S_{i}, E_{i}\right]$ such that in the optimal solution of the continuous relaxation: $\bar{x}_{i t}>0$ if $S_{i} \leq t<E_{i}, \bar{x}_{i E_{i}} \geq 0$, and $\bar{x}_{i t}=0$ for all $t \notin\left[S_{i}, E_{i}\right]$. Moreover, $S_{1}=1, E_{i} \leq S_{i+1}$ for all $i$, and at optimality, $D_{t}$ is strictly decreasing after $E_{n}$, i.e. $D_{E_{n}}>D_{E_{n}+1}>\cdots>D_{T}$. 
This result demonstrates that, when products have equal margins and exponential decays, they are introduced earlier if they stay "fresh" for longer (i.e., they have a higher $\kappa_{i}$ ). Theorem 3 requires a strict ordering of the decay parameters, but when there are several products of the same type (i.e., that share the same decay factor $\kappa_{i}$ ), the staggered product introduction property still applies: there is one optimal solution with such structure. As shown in the following example, the intervals $\left[S_{1}, E_{1}\right],\left[S_{2}, E_{2}\right], \ldots,\left[S_{n}, E_{n}\right]$ may be disjoint.

Example 2 (Non-overlapping intervals). Suppose that $n=2, T=4, r_{1}=r_{2}=\$ 1, \alpha=$ $0.95, v_{0}=1, v_{1}=10, v_{2}=1, \kappa_{1}=0.8$ and $\kappa_{2}=0.4$. The unique solution of the relaxation is to set $\bar{x}_{11}=1, \bar{x}_{1 t}=0$ for $t \neq 1, \bar{x}_{23}=1, \bar{x}_{2 t}=0$ for $t \neq 3$. Thus, $\left[S_{1}, E_{1}\right]=[1,1]$ and $\left[S_{2}, E_{2}\right]=[3,3]$.

When there is a single product type, the solution of the continuous relaxation can be characterized even further, as shown in the next two results. We know from Theorem 3 that there is a cut-off period $E$ after which no product is introduced. Let us denote this cut-off period by $E(\kappa)$ to emphasize its dependence on $\kappa$, since the cut-off period will change with $\kappa$. The existence of $E(\kappa)$ allows simplifying the solution of the KKT conditions above (the details are given in the appendix). We use this fact later to compute our RuleOfThumb heuristic. Moreover, the following result shows that the cut-off period $E(\kappa)$ is non-increasing in $\kappa$.

Proposition 4 (Products with Faster Decay are Introduced over Longer Periods). Assume a single product type and $\alpha_{t}=\alpha^{t-1}$ for all $t$ with $\alpha \leq 1$. Then, $E(\kappa)$ is non-increasing in $\kappa$.

Note that when $\kappa=1$, we have a truly basic product and there is no decay. In this case, it is easy to verify that it is optimal to introduce everything in the first period, that is, $E(1)=1$. Proposition 4 shows that as the product decays faster, with $\kappa$ decreasing to zero, it would gradually be introduced over a longer horizon. In fact, with $\kappa=0$, we have a single-period life cycle, and in this case, $E(0)=T$. As a consequence of the above proposition, we see that basic products are always introduced earlier than fashion-basic products.

The next result shows that the market share under the optimal product introduction is declining over time. The optimal market share in period $t$ is given by $\bar{z}_{t} /\left(v_{0}+\bar{z}_{t}\right)$. The following proposition shows that $\bar{z}_{t}$ is decreases with $t$.

Proposition 5 (Decreasing Optimal Market Share). Assume a single product type and $\alpha_{t}=$ $\alpha^{t-1}$ for all $t$ with $\alpha \leq 1$. In the optimal solution of the continuous relaxation the market share decreases over time, that is, $\bar{z}_{1} \geq \bar{z}_{2} \geq \cdots \geq \bar{z}_{T}$.

The previous results assume that all products have equal margins. With unequal margins the problem is hard to analyze because the continuous relaxation is not even concave. An exception 
is when there is a basic item $j$ with a margin $r_{j} \geq 1$ and all other items have equal margins normalized to 1 . If $v_{0} \geq v_{j}\left(r_{j}-1\right)$, then the problem remains concave and the results in Theorem 3 and Proposition 5 continue to hold. In other words, the optimal solution of the continuous relaxation will follow the staggered introduction property; and if all items $i \neq j$ have the same type, then the optimal market share will be decreasing.

\section{Approximation Algorithms for the $A P P$}

In this section we introduce four approximation algorithms to solve the APP. The first two assume equal margins while the last two are for the general case and allow for products with different margins. Though these are approximate methods, we are able to establish performance guarantees.

5.1 Two Heuristics under Equal Margins. Here we consider the case when all products have the same gross margin, which we normalize to one, i.e., $r_{i}=1, \forall i=1, \ldots, n$. The objective function of the $A P P$ becomes $J:=\sum_{t=1}^{T} \frac{\alpha_{t} z_{t}}{v_{0}+z_{t}}$. Since each fractional term $\frac{\alpha_{t} z_{t}}{v_{0}+z_{t}}$ is increasing in $z_{t}$, it follows that with equal margins it is optimal to introduce all products (this is not necessarily the case with unequal margins). The two heuristics that we introduce next are based on the structure of the continuous relaxation (4) when margins are equal.

5.1.1 Greedy Heuristic. To solve the $A P P$ with equal margins, consider the partial derivative of the objective function $J$ with respect to $x_{i t}$ :

$$
\frac{\partial J}{\partial x_{i t}}=v_{0} \sum_{s=t}^{T} \frac{\alpha_{s} v_{i} \kappa_{i, s-t}}{\left(v_{0}+z_{s}\right)^{2}} .
$$

Motivated by the concavity of the objective function $J$, we propose the following GREEDY heuristic: sequentially assign products to periods (bins), each time selecting the product-period pair with the highest partial derivative (6) among those products that have not been previously assigned. Intuitively, we use the index (6) because this is a measure of the marginal increase in profits, and products are introduced according to this index in a greedy fashion. Note that this heuristic does not require solving the continuous relaxation. Its computation time is $O\left(n^{2} T^{2}\right)$ for general decay patterns but can be computed more efficiently for specific structures such as exponential decay.

While the performance of GREEDY is generally hard to evaluate analytically, when all products have a single-period life cycle, a performance guarantee can be developed. This corresponds to the case where $\kappa_{i, d}=0$ when $d \geq 1$, i.e., products completely lose their attractiveness one period after being introduced. This can be seen as an extreme case of exponential preference decay in which the decay parameter tends to zero for all products, or it can also represent the situation when all 
products have the same planned life cycle, i.e., $\ell_{i}=\ell, \forall i=1, \ldots, n$ (in this case the horizon $T$ should be a multiple of $\ell$ ).

Without loss of generality, as all products have the same margin, we sort the items by decreasing sizes, $v_{1} \geq \ldots \geq v_{n}$, and we assume that $\alpha_{1} \geq \ldots \geq \alpha_{T} \geq 0$. Let $V^{*}$ be the optimal solution to the $A P P$, and let $z_{t}^{*}=\sum_{i=1}^{n} v_{i} x_{i t}^{*}$ be the load for period $t$ in an optimal solution. Note that because $\alpha_{t}$ is decreasing in $t$, it must be true that $z_{t}^{*}$ is also decreasing in $t$. Otherwise, if $z_{t_{1}}^{*}<z_{t_{2}}^{*}$ with $t_{1} \leq t_{2}$, the products allocated to $t_{1}$ and $t_{2}$ could be exchanged and profits would be increased. Hence, we observe that the decreasing market share property that was shown in Proposition 5 for the continuous relaxation holds here for the optimal integral solution of the APP.

With single-period life cycles, the GREEDY heuristic simplifies to the following procedure. First, sort the items in decreasing size. Second, allocate items to periods (bins) in a greedy fashion: place item $i$ in the period with the highest current index $\frac{\alpha_{t}}{\left(v_{0}+z_{t}\right)^{2}}$ where $z_{t}$ is the current load. Clearly the heuristic runs in polynomial time. Moreover, we can provide the following performance guarantee.

Theorem 6. When all products have a single-period life cycle and $\alpha_{t}=1$ for $t=1, \ldots, T$, let $V^{\text {Greedy }}$ be the objective value of the GREEDY solution. Then $1 \leq \frac{V^{*}}{V^{\text {Greedy }}} \leq \frac{9}{8}$.

Remarkably, when there is no seasonality or discounting, Theorem 6 provides a $9 / 8$-approximation for the $A P P$. This result can then be used for more general situations.

Corollary 7. When all products have a single-period life cycle, compute the solution provided by the GREEDY heuristic applied to the problem with $\alpha_{t}=1$ for $t=1, \ldots, T$. Then this heuristic is a $\frac{9}{8} \Lambda$-approximation for the APP, where $\Lambda=\frac{\max _{t} \alpha_{t}}{\min _{t} \alpha_{t}}$.

5.1.2 EarlyEntry Heuristic. The second heuristic, which we call EarlyEntry, is motivated by the staggered entry property shown in Theorem 3 for exponential preference decay. The procedure is simple: solve the continuous relaxation and determine the time window in which each product is released. Then, in the $A P P$, introduce each product at the beginning of its time window. In other words, introduce product $i$ in the earliest period $t$ such that $\bar{x}_{i t}>0$, where $\bar{x}_{i t}$ represents the optimal solution of the continuous relaxation. When the values of $\kappa_{i}$ are large, the EARLYENTRY heuristic generates solutions that are close to the optimum of the APP.

Theorem 8. When all products have exponential preference decay and $\alpha_{t}=\alpha^{t-1}$ for all $t$, let $\left[S_{i}, E_{i}\right]$ denote the release time window in the optimal solution of the continuous relaxation for product $i \in I$. Let $V^{\text {EarlyEntry }}$ be the objective value of the EARLYENTRY solution. Then $1 \leq$ $\frac{V^{*}}{V^{\text {EarlyEntry }}} \leq \max _{i=1, \ldots, n}\left\{\frac{1}{\kappa_{i}^{E_{i}-S_{i}}}\right\}$, where $\kappa_{i}$ is the decay parameter of product $i \in I$. 
This performance bound depends on the actual solution to the continuous relaxation, through the values $S_{i}$ and $E_{i}$. Hence, if $E_{i}=S_{i}$ for all $i$, then the heuristic leads to the optimum, because the relaxation solution is integral. When the number of products is large, the time windows are compressed, so $E_{i}-S_{i}$ is small for all $i$, which leads to a performance bound that only depends on the decay parameters $\kappa_{i}$. The performance bound is tighter when all $\kappa_{i}$ are high. Hence, the EARLYENTRY heuristic is an appropriate complement to GREEDY, which performs well when all $\kappa_{i}$ tend to zero.

5.2 Two Heuristics under General Margins. The two previous heuristics were developed for the case of equal margins. Note that these heuristics provide a feasible solution and an upper bound for the general case if one replaces the margins $r_{i}$ by its maximal value. Here we develop two heuristics that can work with unequal margins directly.

5.2.1 RuleOfThumb Heuristic. This is a simple heuristic that leverages the insights from the paper and can be easily executed in a spreadsheet. The heuristic assumes exponential decay but can be extended to more general decay patterns. The basic idea is to consider all revenue-ordered sets $A_{1}, A_{2}, \ldots, A_{n}$, where for $\ell=1, \ldots, n, A_{\ell}=\{1,2, \ldots, \ell\}$. For each revenue-ordered set $A_{\ell}$, we solve the APP with a single product type. For this product type, the revenue $\bar{r}_{\ell}$ is defined as the weighted average of the $r_{i}$ parameters of the products in $A_{\ell}$, the preference weight $\bar{v}_{\ell}$ is defined as the total preference weight of the products in $A_{\ell}$, and the exponential decay parameter $\bar{\kappa}_{\ell}$ is defined as the power mean of the decay parameters of the products in $A_{\ell}$. The newly constructed product type can be interpreted as a "representative" product of the revenue-ordered set $A_{\ell}$. We then solve the the continuous relaxation of the APP with this newly created product type, and use a greedy heuristic to extrapolate the release date for all products. We repeat this process for each revenue-ordered assortment, and choose the release schedule that gives us the maximum revenue.

We consider revenue-ordered sets because it is common practice in the assortment planning literature and we also found some empirical support as described in Section 6.2. On the other hand, the continuous solution of the APP with a single product type can be fully characterized when decay is exponential (c.f. Section 4.2) so it poses no computational challenge. A formal description of the heuristics is given below.

1. For each $\ell=1, \ldots, n$, consider the revenue-ordered set $A_{\ell}=\{1, \ldots, \ell\}$ and do the following: (a) Let $\bar{r}_{\ell}=\frac{\sum_{i \in A_{\ell}} r_{i} v_{i}}{\sum_{i \in A_{\ell}} v_{i}}, \bar{v}_{\ell}=\sum_{i \in A_{\ell}} v_{i}$, and $\bar{\kappa}_{\ell}=\left(\frac{\sum_{i \in A_{\ell}} \kappa_{i}^{p}}{\left|A_{\ell}\right|}\right)^{\frac{1}{p}}$, where the parameter $p$ is to be determined. 
(b) Solve the continuous relaxation of APP for a single product type with revenue $\bar{r}_{\ell}$, attractiveness $\bar{v}_{\ell}$, and exponential decay with parameter $\bar{\kappa}_{\ell}$. From Propositions 4 and 5 , when there is a single type, the APP relaxation will introduce products during consecutive periods $1, \ldots, E_{\ell}$. We can compute the period $E_{\ell}$ and the optimal loads $z_{t}$ in each period using a simple spreadsheet (details are discussed in the Appendix).

(c) Sort the products in $A_{\ell}$ by decay parameter, from largest to smallest. Start at the top of the list, i.e., with product that has the slowest decay, and introduce products in period $t=1$ while $\bar{r} z_{t}<\sum_{i=1}^{n} \sum_{u=1}^{t} r_{i} v_{i} \kappa_{i}^{t-u} x_{i u}$. Do the same for $t=2, \ldots, T$. This will produce a release schedule for all products.

2. From among the $n$ product release schedules, choose the one that gives the highest revenue.

In our experiments, when we computed the power mean to determine $\bar{\kappa}_{\ell}$, we chose $p=8$ because it gave the best results in our numerical experiments but the heuristic works for any value of $p$. Also, $\bar{\kappa}$ is greater than the arithmetic mean because it gives more weight to the higher $\kappa_{i}$ 's, which is consistent with the insight that products with slower decay should be introduced earlier. Note that the above heuristic introduces the products in $A_{\ell}$ in a staggered fashion dictated by the single-product relaxation. This is done for all values of $\ell$.

5.2.2 Randomized Heuristic. Here we follow the randomized approach that has been used in other combinatorial problems (see Raghavan and Thompson 1987). We construct this heuristic by noting that the constraint (2) in the APP allows giving a probabilistic interpretation to the solution of the continuous relaxation. Let $\left(\bar{x}_{i t}: i \in I, t=1, \ldots, T\right)$ denote the solution to the continuous relaxation problem and let $\bar{V}$ be its objective value. Let $Q_{i}$ denote a random variable that represents the time period when product $i \in I$ is introduced. The probability distribution of $Q_{i}$ is given by the solution to the continuous relaxation. To be precise, let $\operatorname{Pr}\left(Q_{i}=t\right)=\bar{x}_{i t}, \forall i \in I, t=1, \ldots, T$. Since with unequal margins constraint (2) might not be binding, we consider a fictitious period $T+1$ to represent the non-release option with probability mass $\operatorname{Pr}\left(Q_{i}=T+1\right)=1-\sum_{t=1, \ldots, T} \bar{x}_{i t}, \forall i \in I$. Let $f\left(Q_{1}, \ldots, Q_{n}\right)$ denote the (random) profit associated with the random vector $\left(Q_{1}, \ldots, Q_{n}\right)$, i.e.,

$$
f\left(Q_{1}, \ldots, Q_{n}\right):=\sum_{t=1}^{T} \alpha_{t} \frac{\sum_{u=1}^{t} \sum_{i=1}^{n} r_{i} \kappa_{i, t-u} v_{i} \mathbb{1}_{\left[Q_{i}=u\right]}}{v_{0}+\sum_{u=1}^{t} \sum_{i=1}^{n} \kappa_{i, t-u} v_{i} \mathbb{1}_{\left[Q_{i}=u\right]}},
$$

where $\mathbb{1}_{A}$ is the indicator function for event $A$. The following theorem gives a performance guarantee associated with the random vector $\left(Q_{1}, \ldots, Q_{n}\right)$.

Theorem 9. Let $\rho:=\frac{v_{0}}{v_{0}+\max _{j=1, \ldots, n} v_{j}}$. Then, $1 \leq \frac{V^{*}}{\mathbb{E}\left[f\left(Q_{1}, \ldots, Q_{n}\right)\right]} \leq \frac{\bar{V}}{\mathbb{E}\left[f\left(Q_{1}, \ldots, Q_{n}\right)\right]} \leq \frac{1}{\rho}$. 
Based on the probabilistic interpretation described above, we define a RANDOMIZED heuristic that consists in sampling a fixed number of solutions for the APP according to the probability distribution of $\left(Q_{1}, \ldots, Q_{n}\right)$ and keeping the best one. Note that the inequality $\mathbb{E}\left[f\left(Q_{1}, \ldots, Q_{n}\right)\right] \geq$ $\rho \bar{V}$ in Theorem 9 ensures that sampling the distribution of $\left(Q_{1}, \ldots, Q_{n}\right)$ will eventually yield a solution with an objective value greater than $\rho \bar{V}$, where $\rho=\frac{v_{0}}{v_{0}+\max _{j=1, \ldots, n} v_{j}}$. This solution is guaranteed to be within percentage $1-\rho$ of the optimal value $V^{*}$. Clearly, the performance guarantee for the RANDOMIZED heuristic is tighter when $\rho$ is larger, which occurs when the outside option has significant weight $v_{0}$ vis-a-vis the attractiveness of the products the firm can offer. This situation usually occurs when the market is competitive and customers have many other alternatives where to purchase. For instance, if $v_{0} \geq v_{i}, \forall i \in I$, which is a common assumption found in the literature (e.g., Allon et al. 2010), then the RANDOMIZED solution is within $50 \%$ of the optimum.

As mentioned in Section 4, solving the continuous relaxation with unequal margins is challenging and one of the prevalent methods used is b\&b. Given the substantial computational effort that is required, in practice the $b \& b$ procedure is usually terminated when a feasible solution is found that satisfies a sub-optimality tolerance. If that is the case, the performance guarantee of the RANDOMIZED heuristic can be modified according to the following corollary.

Corollary 10. Let $\left(\widetilde{x}_{i t}: i \in I, t=1, \ldots, T\right)$ represent the incumbent solution in the branchand-bound procedure and let $\widetilde{J}$ be its objective value. Let $\widetilde{V}$ be the current upper bound and let $\Gamma:=(\widetilde{V}-\widetilde{J}) / \widetilde{V}$ be the sub-optimality gap. Then, the RANDOMIZED heuristic using probability weights $\widetilde{x}_{i t}$ guarantees that $1 \leq \frac{V^{*}}{\mathbb{E}\left[f\left(Q_{1}, \ldots, Q_{n}\right)\right]} \leq \frac{1}{(1-\Gamma) \rho}$, where $\rho$ is defined as in Theorem 9 .

\section{Numerical Study}

Our numerical study has two parts. First, in Section 6.1 we describe in detail a small illustrative example to shed further light on the problem, its structure, and the merits of the approximation algorithms. Then in Section 6.2 we consider a realistic sized problem instance using data on sales and release dates of women handbags from a Spanish accessories retailer. Note that, as a benchmark, we report the sub-optimality gap of the solution that introduces all products in the first period, which we denote AlLEarly. For our study, the continuous relaxation was coded in GAMS and was solved using the commercial non-linear solver CONOPT2. The approximation algorithms were coded in Perl. The running times in all cases did not surpass a few minutes.

6.1 Small Illustrative Examples. Here we consider an instance with ten periods and four products that have equal margins and attractiveness: $v_{1}=1, v_{2}=2, v_{3}=3, v_{4}=100$, so there 
are three minor items and one "fat" product. We assume exponential decay with parameters: $\kappa_{1}=0.9, \kappa_{2}=0.6, \kappa_{3}=0.5, \kappa_{4}=0.4$. The outside option weighs $v_{0}=1$ and there is no discounting.

Table 1 show the results for this small instance. The objective value of the different solutions is reported on the left hand side of the table, while the actual solution is shown to the right. The first entry of the table is the optimal solution to the continuous relaxation, which has a objective value of 9.457. This value is a very loose upper bound for the $A P P$ due to the presence of the fat item. Therefore, we did ten b\&b steps - branching on the release period for product 4 - which reduced the upper bound to 8.269. This result is shown as the second entry in Table 1. The third entry is the optimal integer solution, which we were able to find by exhausting the b\&b tree for this small example. Though there is a $0.54 \%$ gap between the optimal integer solution and the partial b\&b upper bound, we assess the sub-optimality of the approximation algorithms with respect to the latter emulating the fact that the true integer optimum would be computationally prohibitive to find for any real-size instance. The remaining three entries in Table 1 report the performance of the heuristics described in Section 5.

\begin{tabular}{|c|c|c|c|c|c|c|c|c|c|c|c|c|}
\hline \multirow[t]{2}{*}{ Algorithm } & \multirow{2}{*}{$\begin{array}{c}\text { Objective } \\
\text { (\% gap) }\end{array}$} & \multicolumn{11}{|c|}{ Solution $x_{i t}$} \\
\hline & & $i, t$ & 1 & 2 & 3 & 4 & 5 & 6 & 7 & 8 & 9 & 10 \\
\hline \multirow{4}{*}{$\begin{array}{c}\text { Continuous } \\
\text { relaxation }\end{array}$} & \multirow[t]{4}{*}{9.457} & 1 & 1.000 & 0.000 & 0.000 & 0.000 & 0.000 & 0.000 & 0.000 & 0.000 & 0.000 & 0.000 \\
\hline & & 2 & 1.000 & 0.000 & 0.000 & 0.000 & 0.000 & 0.000 & 0.000 & 0.000 & 0.000 & 0.000 \\
\hline & & 3 & 1.000 & 0.000 & 0.000 & 0.000 & 0.000 & 0.000 & 0.000 & 0.000 & 0.000 & 0.000 \\
\hline & & 4 & 0.120 & 0.096 & 0.099 & 0.102 & 0.103 & 0.104 & 0.104 & 0.105 & 0.105 & 0.063 \\
\hline \multirow{4}{*}{$\begin{array}{l}\text { Partial b\&b } \\
\text { upper bound }\end{array}$} & \multirow[t]{4}{*}{8.269} & 1 & 0.000 & 0.000 & 0.000 & 0.000 & 1.000 & 0.000 & 0.000 & 0.000 & 0.000 & 0.000 \\
\hline & & 2 & 0.000 & 0.000 & 0.000 & 0.000 & 0.000 & 0.685 & 0.315 & 0.000 & 0.000 & 0.000 \\
\hline & & 3 & 0.000 & 0.000 & 0.000 & 0.000 & 0.000 & 0.000 & 0.176 & 0.391 & 0.413 & 0.021 \\
\hline & & 4 & 1.000 & 0.000 & 0.000 & 0.000 & 0.000 & 0.000 & 0.000 & 0.000 & 0.000 & 0.000 \\
\hline \multirow[t]{4}{*}{ OPTIMAL } & \multirow{4}{*}{$\begin{array}{c}8.224 \\
(0.54 \%)\end{array}$} & 1 & 0.000 & 0.000 & 0.000 & 0.000 & 1.000 & 0.000 & 0.000 & 0.000 & 0.000 & 0.000 \\
\hline & & 2 & 0.000 & 0.000 & 0.000 & 0.000 & 0.000 & 1.000 & 0.000 & 0.000 & 0.000 & 0.000 \\
\hline & & 3 & 0.000 & 0.000 & 0.000 & 0.000 & 0.000 & 0.000 & 0.000 & 1.000 & 0.000 & 0.000 \\
\hline & & 4 & 1.000 & 0.000 & 0.000 & 0.000 & 0.000 & 0.000 & 0.000 & 0.000 & 0.000 & 0.000 \\
\hline \multirow[t]{4}{*}{ GREEDY } & \multirow{4}{*}{$\begin{array}{c}8.198 \\
(0.85 \%)\end{array}$} & 1 & 0.000 & 0.000 & 0.000 & 0.000 & 1.000 & 0.000 & 0.000 & 0.000 & 0.000 & 0.000 \\
\hline & & 2 & 0.000 & 0.000 & 0.000 & 0.000 & 0.000 & 0.000 & 1.000 & 0.000 & 0.000 & 0.000 \\
\hline & & 3 & 0.000 & 0.000 & 0.000 & 0.000 & 0.000 & 0.000 & 0.000 & 1.000 & 0.000 & 0.000 \\
\hline & & 4 & 1.000 & 0.000 & 0.000 & 0.000 & 0.000 & 0.000 & 0.000 & 0.000 & 0.000 & 0.000 \\
\hline \multirow{4}{*}{$\begin{array}{c}\text { EARLYENTRY } \\
\text { RuLEOFTHUMB } \\
\text { AlLEARLY }\end{array}$} & \multirow{4}{*}{$\begin{array}{c}6.836 \\
(17.33 \%)\end{array}$} & 1 & 1.000 & 0.000 & 0.000 & 0.000 & 0.000 & 0.000 & 0.000 & 0.000 & 0.000 & 0.000 \\
\hline & & 2 & 1.000 & 0.000 & 0.000 & 0.000 & 0.000 & 0.000 & 0.000 & 0.000 & 0.000 & 0.000 \\
\hline & & 3 & 1.000 & 0.000 & 0.000 & 0.000 & 0.000 & 0.000 & 0.000 & 0.000 & 0.000 & 0.000 \\
\hline & & 4 & 1.000 & 0.000 & 0.000 & 0.000 & 0.000 & 0.000 & 0.000 & 0.000 & 0.000 & 0.000 \\
\hline \multirow[t]{4}{*}{ RANDOMIZED } & \multirow{4}{*}{$\begin{array}{c}8.067 \\
(2.50 \%)\end{array}$} & 1 & 1.000 & 0.000 & 0.000 & 0.000 & 0.000 & 0.000 & 0.000 & 0.000 & 0.000 & 0.000 \\
\hline & & 2 & 1.000 & 0.000 & 0.000 & 0.000 & 0.000 & 0.000 & 0.000 & 0.000 & 0.000 & 0.000 \\
\hline & & 3 & 1.000 & 0.000 & 0.000 & 0.000 & 0.000 & 0.000 & 0.000 & 0.000 & 0.000 & 0.000 \\
\hline & & 4 & 0.000 & 0.000 & 0.000 & 0.000 & 1.000 & 0.000 & 0.000 & 0.000 & 0.000 & 0.000 \\
\hline
\end{tabular}

Table 1: Small numerical example with 4 products and 10 periods. The gaps are with respect to the upper bound (8.269).

As expected, the continuous relaxation solution has the staggered entry property shown in Theorem 3, with the first three items released only in the first period while the fat item is released 
continuously throughout the horizon, which makes the solution very fractional. In general, we observed this behavior whenever there was a fat item, i.e., a product that was significantly more attractive than the rest. In those cases, the optimal continuous solution has a large integrality gap. For the purpose of this example, we reduced the gap through b\&b, first partially and then exhaustively. The idea of doing partial b\&b is to show that typically branching on the fat item(s) is enough to narrow the integrality gap to a reasonable level, which can be very useful in practice.

In contrast to the continuous relaxation, the optimal integral solution has the fat item as the sole product introduced in the first period despite the fact that it has the fastest decay. The remaining products are introduced only once the attractiveness of the fat item has vanished. The GREEDY solution has this same structure - i.e., the fat item first and the rest later - and it achieves the best performance among the solutions considered. In fact, except for product 2, the GREEDY solution introduces all items optimally, which shows that the partial derivative (6) is indeed a good optimality indicator. The EARLYENTRY and RANDOMIZED solutions are based on the continuous relaxation, which we know has a large integrality gap. In particular, the EARLYENTRY solution, which coincides with RuleOfThumb and AllEarly here, is misguided by the fractional values of the fat item, so in this case it has very poor performance. The RANDOMIZED algorithm is able to detect that it would be better to introduce the fat item later, so it has a much better performance, though in terms of structure it is exactly the opposite from the optimal integer solution. Note that once the release of the fat item is fixed by doing partial b\&b, the continuous solution for the remaining items also has the staggered entry property. Hence, the performance of EARLYENTRY and RANDOMIZED usually can be improved by using the partial b\&b solution as the input instead of the original continuous solution.

To further illustrate the previous remarks, we generated a random instance in which a planner has to introduce 52 products in the same number of periods. We assumed no discounting or seasonality, and we generated the attractiveness $v_{i}$ from a uniform $[0,1]$ distribution for all items except one, which had an attractiveness of 10 . The idea was to represent the situation in which there was a big disparity among products and one was substantially more appealing. For the outside option, we considered three different values of $v_{0}(0.5,1$ and 101). The sub-optimality gaps of the approximation algorithms are shown in Table 2. The first three rows assume exponential decay - with the $\kappa_{i}$ parameters again generated randomly, between 0 and 1 - and the last three rows correspond to the case when all products have a single-period life cycle, i.e., when $\kappa_{i} \rightarrow 0, \forall i \in I$. To tighten the upper bound, we did partial b\&b branching on the "fat" item, as in the small example described above. With exponential decay, all the approximation algorithms continued to 
perform very well. In contrast, when all products had a single-period life cycle and the weight of the outside good was low, the performance of the algorithms was very poor except for GREEDY, which as expected achieved sub-optimality gaps within $1 / 8$ given by the performance guarantee in Theorem 6. Note that AlLEARLY performed badly, especially with single-period life cycles, and it was systematically outperformed by all four approximation algorithms. This suggests that there is significant value in properly timing the introduction of products. Finally, note that the performance of the heuristics was similar even when products were homogeneous, i.e., when there was no fat item.

\begin{tabular}{|cc|c|ccccc|}
\hline$v_{0}$ & Life cycle & Upper bound & AllEARLY & GREEDY & EARLYENTRY & RulEOfThumB & RANDOMIZED \\
\hline \hline 0.5 & 52 & 44.06 & $10.912 \%$ & $\mathbf{0 . 2 6 5 \%}$ & $1.420 \%$ & $3.996 \%$ & $0.795 \%$ \\
1 & 52 & 38.25 & $16.312 \%$ & $\mathbf{0 . 3 8 5 \%}$ & $1.971 \%$ & $5.386 \%$ & $1.108 \%$ \\
101 & 52 & 1.47 & $7.606 \%$ & $\mathbf{0 . 0 6 7 \%}$ & $0.274 \%$ & $0.884 \%$ & $0.098 \%$ \\
0.5 & 1 & 27.29 & $96.383 \%$ & $\mathbf{9 . 5 6 9 \%}$ & $25.306 \%$ & $32.831 \%$ & $15.417 \%$ \\
1 & 1 & 18.67 & $94.779 \%$ & $\mathbf{7 . 6 4 8 \%}$ & $16.856 \%$ & $24.874 \%$ & $9.144 \%$ \\
101 & 1 & 0.37 & $25.058 \%$ & $\mathbf{0 . 1 2 3 \%}$ & $0.208 \%$ & $0.413 \%$ & $\mathbf{0 . 1 2 3 \%}$ \\
\hline
\end{tabular}

Table 2: Sub-optimality gaps for a randomly generated instance with 52 periods and 52 products including a "fat" item. AlLEARLY is the solution that introduces all products in the first period. The smallest sub-optimality gap in each row is in boldface.

6.2 Large Instances with Unequal Margins. In order to test our methods on real-size instances, we obtained data from a retailer based in Spain that sells clothing accessories such as belts, hats, scarfs, handbags, etc. The company plans its assortment at the category level in sixmonth cycles and for a network of roughly 60 domestic stores. Currently, the timing of product releases are decided through an ad-hoc manual process based on experience and informed judgement. For our numerical study, we focused on the handbags category because it had enough product variety to make the problem interesting and also there was two years worth of data divided in four seasons from Fall-Winter 2009 to Spring-Summer 2011. The data did not include clearance sales since product introductions only occurred during the regular season. The tables here below are for Fall-Winter 2010 but the results were similar for the other seasons. We assumed exponential decay and used least squares to estimate the parameters $v_{i}$ and $\kappa_{i}$ by fitting the terms $\sum_{t=1}^{T} \frac{v_{i} \sum_{u=1}^{t} \kappa_{i}^{t-u} x_{i u}}{v_{0}+\sum_{j=1}^{n} v_{j} \sum_{u=1}^{t} \kappa_{j}^{t-u} x_{j u}}$ to the actual sales participation observed for each product $i \in \mathcal{I}$. We did not have enough information to estimate the weight of the outside option so we assumed $v_{0}=1$ and then preformed a sensitivity analysis on its value. We did not consider a discount factor because it was not part of the company's current practice. Our estimation provided a reasonable fit, with a relative error of $29 \%$ stemming from a standard error of 0.049 percentage points over an average market share of $0.168 \%$. 
The Fall-Winter 2010 season had 150 products that were introduced over 20 weeks. The average attractiveness of the handbags was 0.001 , and there were no major variations across products: the largest item accounted for only $8 \%$ of the sum of attractiveness of the 150 products; and the $20 \%$ biggest SKUs accounted for $60 \%$ of the sum of attractiveness. The decay parameters ranged from 0.294 to 0.999 , with an average value of $0.777 ; 24$ products $(16 \%$ of all) were truly basics with $\kappa_{i}=1$, while 58 (39\% of all) could be considered fashion-basic items, with $\kappa_{i}<0.7$. The product margins were unequal and increased ten times for the lowest margin to the highest (the actual values cannot be disclosed). Due to the unequal margins, the continuous relaxation of the model was not concave so the solution we obtained from GAMS had to be regarded as a local optimum. For this reason, we do not report sub-optimality gaps for the heuristics but instead we show the improvement with respect to the solution that was implemented by the company.

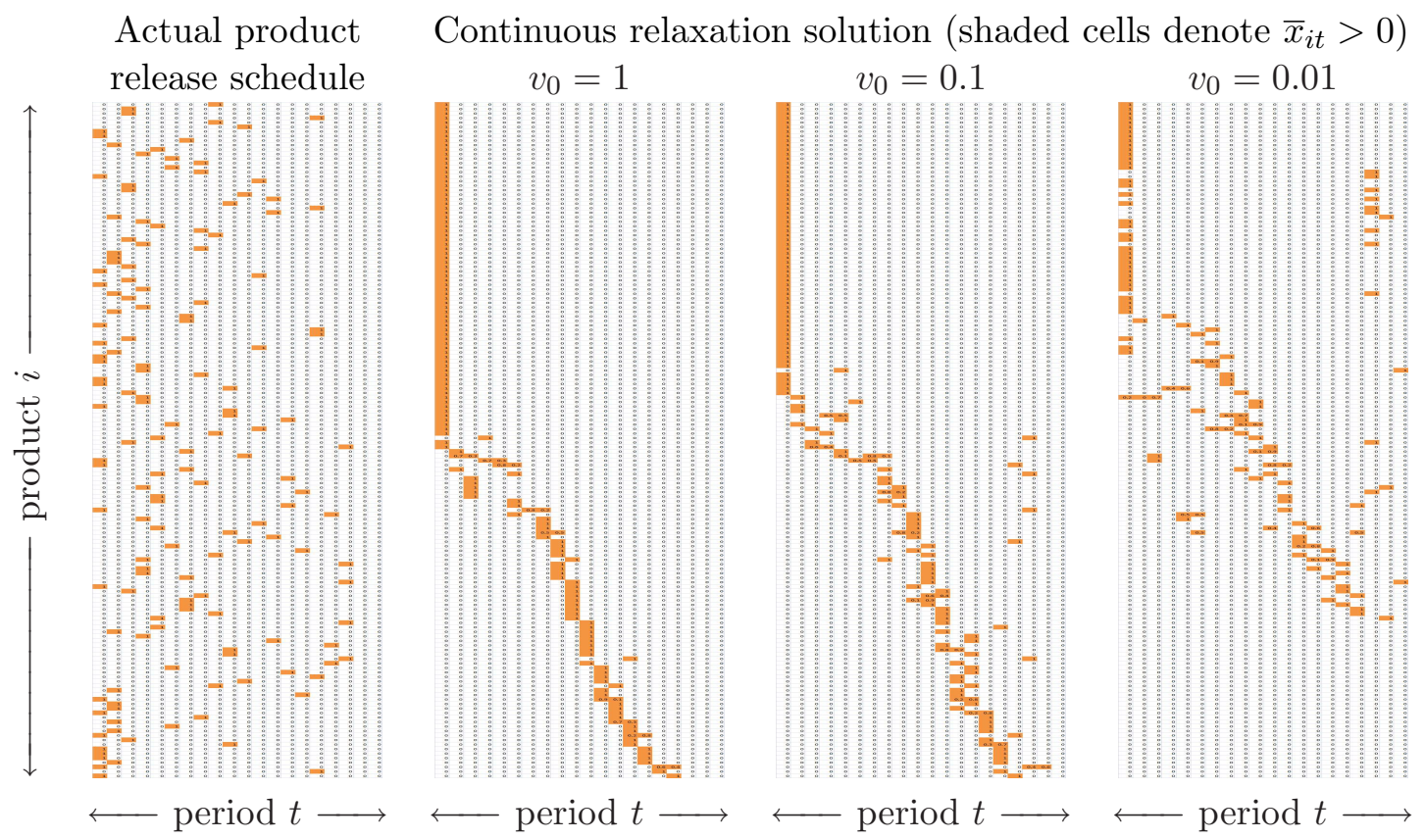

Figure 1: Matrix representation of product release decisions. Positive entries $\left(x_{i t}>0\right)$ are shaded. The leftmost pane shows the actual release schedule in the Fall-Winter 2010 season. The other three panes show the continuous relaxation solution for different values of $v_{0}$. In each matrix, the rows (products) with a positive entry are sorted by decay parameter, from largest to smallest.

Figure 1 shows the solution implemented by the company (leftmost pane) and the solution of the continuous relaxation for three different values of $v_{0}$. Note that the value of $v_{0}$ is directly related to the company's market share and as a result can usually be estimated with good accuracy; fortunately, our tests indicate that the optimal policies perform well even when $v_{0}$ is misspecified. Products are sorted by margins and then those that are released in each solution are resorted by 
decay parameter. Our first observation from Figure 1 is that the firm's solution looks scattered, which shows that the firm did not account for preference decay in its product release decisions. The second observation is that when $v_{0}=0.01$ the continuous relaxation solution suggests not introducing all products. More interestingly, the set of products introduced (in some period) is revenue-ordered. In other words, only the products with the highest margins are released. This empirical fact prevailed for all the values of $v_{0}$ that we tested. The third observation is that, when the weight of the outside option is not too low, the solution of the continuous relaxation resembles the staggered entry property of the equal margin case (c.f. Theorem 3). In additional experiments we observed that when $v_{0}$ tended to zero, the staggered entry property was lost and products were released exclusively based on their margins.
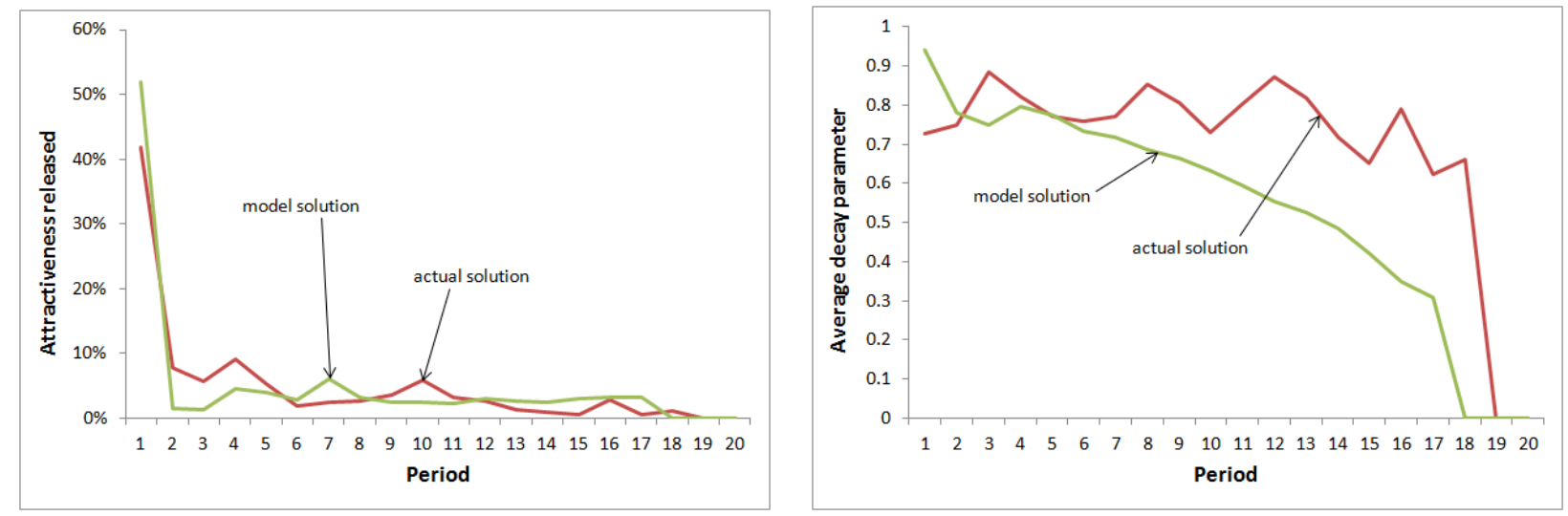

Figure 2: Actual release schedule versus continuous relaxation solution with $v_{0}=1$. The left graph shows the total attractiveness of the products released in each period as a percentage of the season total. The right graph shows the average decay parameter of the products released in each period.

Figure 2 further examines the actual release schedule implemented by the company versus the continuous relaxation solution with $v_{0}=1$. From the left graph one can see that both solutions look similar in terms of attractiveness "released" in each period. However, the right graph shows that average decays are very different. The average decay of the new products in the actual solution remains between 0.7 and 0.9 during most part of the season whereas in the model-based solution it starts high at 0.94 and drops quickly, which is a reflection of the staggered entry shown in Figure 1. This leads to a significant difference in performance as shown next.

Table 3 shows the improvement in profits of our heuristics, when compared to the release dates implemented by the company, for different values of $v_{0}$. For completeness, we report the performance of GREEDY and EARLYENTRY though they were developed for the equal margin case. As before, we also report AllEarly as a benchmark. In all cases, EARlyEntry and RANDOmized 


\begin{tabular}{|c|ccccc|}
\hline$v_{0}$ & ALlEARly & GREEDY & EARLYEnTRY & RULEOFThumB & RANDOMIZED \\
\hline \hline 1 & $17.27 \%$ & $18.52 \%$ & $\mathbf{1 8 . 5 3 \%}$ & $17.37 \%$ & $\mathbf{1 8 . 5 3 \%}$ \\
0.1 & $7.17 \%$ & $12.74 \%$ & $\mathbf{1 2 . 9 6 \%}$ & $10.82 \%$ & $\mathbf{1 2 . 9 6 \%}$ \\
0.01 & $0.72 \%$ & $27.20 \%$ & $\mathbf{2 8 . 7 1 \%}$ & $24.22 \%$ & $\mathbf{2 8 . 7 1 \%}$ \\
0.001 & $0.32 \%$ & $86.00 \%$ & $\mathbf{8 9 . 7 0 \%}$ & $85.52 \%$ & $\mathbf{8 9 . 7 0 \%}$ \\
\hline
\end{tabular}

Table 3: Improvement with respect to implemented solution for different values of $v_{0}$. The best percentage improvement in each row is in boldface.

had the best improvement, which hints that the release time windows of the continuous relaxation can provide good guidance even with unequal margins. The GREeDY algorithm performed well when $v_{0}$ was sufficiently large, but otherwise did slightly worse than EARLYENTRY and RANDOMIZED, which was no surprise because it was constructed with equal margins in mind. Our RuleOfThumb did reasonably well in all instances, which means that simple rules based on the structure of the problem can yield significant gains. Finally, ALLEARLY was systematically outperformed but it came close when $v_{0}$ was large. This was expected since for large $v_{0}$ the firm has a very small market share: as a result, there is little cannibalization across products and it is optimal to introduce all products at the beginning. In contrast, when $v_{0}$ is very small, the firm has a large market share and cannibalization becomes an important issue. In that case, AlLEARLY performs badly for two reasons: first, it introduces all products, while the rest of the heuristics may not do so; and second, it introduces them all at once, which exacerbates cannibalization in early periods, while the rest of the heuristics time the product releases to avoid that.

6.3 Choice of Heuristics. Our experiments provide several insights regarding when each heuristic is most appropriate. First, we observe that when a firm's market share is very small, there is little value in optimizing over product introduction times: products should all be introduced at the beginning of the season, as with AllEarly, because there is little cannibalization across products. In contrast, when a firm's market share is larger, then spreading product introductions over the season is most valuable and our heuristics become relevant. Two situations are then possible: when there are a few "fat" products (with very large attractiveness), then GREEDY is able to correctly place these large items in the season, and thus performs very well; when there are many small products, then EARLYENTRY and RANDOMIZED, guided by the relaxation, perform best. Furthermore, we develop RuleOfThumb as a heuristic that does not require optimization and has the same spirit as EARLYENTRY and RANDOMIZED; it also has an acceptable performance compared to those. Finally, it is worth mentioning that we tested the four heuristic in a variety of other settings: with non-exponential decays, different demand classes (see $\S 7.3$ below), and when $v_{0}$ is misspecified, and we observed similar results. 


\section{Extensions}

We discuss here several extensions of our model. In the next section, we consider constraints on budget and shelf space. Then, in the next two sections, we extend the APP to allow product removals, and to allow for heterogenous populations.

7.1 Budget or Shelf-Space Constraint. One of the implicit assumptions in the original model formulation is that the number of products introduced in a given period is only restricted by how many are available. In practice, it could occur that there is limitation that prevents the firm from releasing more than a certain number of new items. For instance, category managers in apparel some times are given a budget and there is an operational cost associated to each new introduction, so they have to be more selective and not all products are released. This situation can be included in the model by considering the constraint $\sum_{t=1}^{T} \sum_{i=1}^{n} x_{i t} \leq N$ or a variation of it, where $N$ represents the total number of products that can be introduced (if the budget is $B$ and the introduction cost is $c$, then $N=\lfloor B / c\rfloor)$. Another reason that could limit the number of products is some sort of shelf space constraint, which could be incorporated in the model by imposing the inequality $\sum_{i=1}^{n} \sum_{u=1}^{t} S_{i, t-u} x_{i u} \leq S, \forall t \geq 1$, where $S$ is the available space for display and $S_{i, l}$ is the space that product $i$ takes $l$ periods after its introduction.

Adding a budget or shelf space constraint is likely to affect the structure of the problem. However, it is interesting to notice that the fluid interpretation of the continuous relaxation and Theorem 2 remain valid. With equal margins, the GREEDY and EARLYENTRY can be modified to accommodate the additional constraint but the performance guarantees in Section 5.1 are lost, or at least they would require a different proof. In contrast, with general margins, the performance bound for the RANDOMIZED algorithm in Theorem 9 continues to hold. Generating the randomized solution would have to incorporate the product introduction limitation, but this can be achieved by sampling the variables sequentially and resolving the $A P P$ each time a variable is fixed to update the probability distributions, or alternatively one can try to adapt the dependent rounding algorithms described in Bertsimas et al. (1999).

7.2 Product Removals. We can extend the APP to allow for product removals. All assumptions remain the same except that now after a product has been introduced, the planner can decide to remove it once and for all. The fact that a product cannot be reintroduced is consistent with our irrevocability assumption and with the usual practice at most retailers. Note that this model extension only makes sense with unequal margins since when all margins are identical (and no budget or shelf-space constraints are present), it is never optimal to remove a product. 
For $i \in I$ and $t=1, \ldots, T$, let $x_{i t}$ and $y_{i t}$ be the binary decision variables defined by:

$$
\begin{aligned}
& x_{i t}= \begin{cases}1 & \text { if product } i \text { is introduced at the beginning of period } t \\
0 & \text { otherwise. }\end{cases} \\
& y_{i t}= \begin{cases}1 & \text { if product } i \text { is removed at the end of period } t \\
0 & \text { otherwise. }\end{cases}
\end{aligned}
$$

The APP with product removals can be formulated as the following binary optimization problem.

$$
\begin{array}{lll}
(A P P-R) & V^{*}=\max _{t=1}^{T} \alpha_{t} \sum_{i=1}^{n} r_{i} \times\left(\frac{v_{i} \sum_{u=1}^{t} \kappa_{i, t-u} z_{i, u, t}}{v_{0}+\sum_{j=1}^{n} v_{j} \sum_{u=1}^{t} \kappa_{j, t-u} z_{i, u, t}}\right) \\
\text { s.t. } & & \forall i \in I, \\
\sum_{t=1}^{T} x_{i t} \leq 1 & \forall i \in I, \\
\sum_{t=1}^{T} y_{i t} \leq 1 & \forall i \in I, t=1,2, \ldots, T \\
\sum_{s=1}^{t} x_{i s} \geq \sum_{s=1}^{t} y_{i s} & \forall i \in I, 1 \leq u \leq t \leq T \\
z_{i, u, t} \leq x_{i u} & \forall i \in I, 1 \leq u \leq t \leq T \\
z_{i, u, t} \leq 1-\sum_{s=1}^{t-1} y_{i s} & \forall i \in I, 1 \leq u \leq t \leq T \\
z_{i, u, t} \geq x_{i u}-\sum_{s=1}^{t-1} y_{i s} & \forall i \in I, t=1,2, \ldots, T \\
x_{i t} \in\{0,1\} y_{i t} \in\{0,1\} & & \forall i \in I, 1 \leq u \leq t \leq T
\end{array}
$$

The objective function (8) is the sum of the discounted profits over all products from each of the $T$ periods in the season. The expression in the parentheses represents the market share of product $i$ in period $t$. The variable $z_{i, u, t}$ has the following interpretation: $z_{i, u, t}=1$ if and only if product $i$ is introduced in period $u$ and is still active in period $t$. To see this, suppose that we introduce product $i$ in period $b$ and remove it at the end of period $e$, with $b \leq e$; that is, $x_{i b}=y_{i e}=1$. Then, the constraints (12), (13), and (14) imply that

$$
x_{i u}-\sum_{s=1}^{t-1} y_{i s} \leq z_{i, u, t} \leq \min \left\{x_{i u}, 1-\sum_{s=1}^{t-1} y_{i s}\right\} \quad 1 \leq u \leq t \leq T .
$$

Since $x_{i b}=y_{i e}=1$,

$$
\min \left\{x_{i u}, 1-\sum_{s=1}^{t-1} y_{i s}\right\}= \begin{cases}0 & \text { if } u \neq b \text { or } t \geq e+1 \\ 1 & \text { if } u=b \text { and } t \leq e\end{cases}
$$

which implies, by constraint (16), that $z_{i, u, t}=0$ when $u \neq b$ or $t \geq e+1$. Now, when $u=b$ and $t \leq e$, we have that $x_{i u}-\sum_{s=1}^{t-1} y_{i s}=1-0=1$, and thus,

$$
z_{i, u, t}=\left\{\begin{array}{ll}
0 & \text { if } u \neq b \text { or } t \geq e+1, \\
1 & \text { if } u=b \text { and } t \leq e
\end{array},\right.
$$


which is the desired result. Moreover, for $t \in\{b, b+1, \ldots, e\}, \sum_{u=1}^{t} \kappa_{i, t-u} z_{i, u, t}=\kappa_{i, t-b}$, which gives the correct decay factor in the objective function.

Let us now discuss the remaining constraints. Constraint (15) imposes the binary requirement on the introduction and removal dates. Constraints (9) and (10) ensure that each product is introduced and removed, respectively, at most once. Constraint (11) ensures that a product is always introduced before it is removed. Our formulation allows for a product to be displayed in the store for just one period, corresponding to the case where $x_{i t}=y_{i t}=1$ for some $t$.

It is possible to construct small instances in which the planner does (slightly) better by being allowed to remove products. However, for the real-size instances of Section 6.2 we did not see any improvement because the local optimum found by the commercial solver did not remove any products. The reason for this is that, when a low-margin product could be a candidate for removal, its attractiveness has usually decayed so much that product cannibalization is no longer an issue, and thus it is best not to remove it.

7.3 Customer Heterogeneity. In this section, we describe the APP problem with mixture of logits and show that the fluid approximation result continues to hold. The mixture of logits may be an appropriate model when the population consists of heterogenous segments, but we do not know the segment associated with each customer entering our store. Suppose that our customer base consists of $G$ distinct segments indexed by $g=1,2, \ldots, G$. For each group $g$, let $\boldsymbol{v}^{g}=\left(v_{0}^{g}, v_{1}^{g}, \ldots, v_{n}^{g}\right)$ denote the initial preference weights for the no-purchase option and for each of the $n$ products. Once a product is introduced in the store, its rate of decay will depend on both the duration it has been in the store and the customer segments. For each segment $g$, let $\left\{\kappa_{i, d}^{g}: i=1, \ldots, n, d=0,1, \ldots\right\}$ denote the corresponding decay parameters. Also, let $\zeta^{g} \in[0,1]$ denote the fraction of customers in group $g$, with $\sum_{g=1}^{G} \zeta^{g}=1$. Then, we have the following APP:

$$
\begin{array}{rr}
(A P P-M) \quad V^{*}=\max & \sum_{t=1}^{T} \alpha_{t} \sum_{i=1}^{n} r_{i} \sum_{g=1}^{G} \zeta^{g} \times\left(\frac{v_{i}^{g} \sum_{u=1}^{t} \kappa_{i, t-u}^{g} x_{i u}}{v_{0}^{g}+\sum_{j=1}^{n} v_{j}^{g} \sum_{u=1}^{t} \kappa_{j, t-u}^{g} x_{j u}}\right) \\
\text { s.t. } \quad \sum_{t=1}^{T} x_{i t} \leq 1 & \forall i \in I, \\
x_{i t} \in\{0,1\} & \forall i \in I, t=1, \ldots, T,
\end{array}
$$

where the expression in the parentheses in the objective function represents the market share of product $i$ in period $t$ from customers in segment $g$. The corresponding continuous optimization 
problem is given by:

$\bar{V}=\max \left\{\sum_{t=1}^{T} \alpha_{t} \sum_{i=1}^{n} r_{i} \sum_{g=1}^{G} \zeta^{g} \times\left(\frac{v_{i}^{g} \sum_{u=1}^{t} \kappa_{i, t-u}^{g} x_{i u}}{v_{0}^{g}+\sum_{j=1}^{n} v_{j}^{g} \sum_{u=1}^{t} \kappa_{j, t-u}^{g} x_{j u}}\right) \mid \sum_{t=1}^{T} x_{i t} \leq 1 \forall i, \quad x_{i t} \geq 0 \forall i, t\right\}$, and let $\left\{\bar{x}_{i t}: i=1, \ldots, n, t=1, \ldots, T\right\}$ be an optimal solution to the above problem.

It can be shown that the continuous relaxation can be interpreted as a fluid approximation similar to the non-mixture case (c.f. Theorem 2). We do not expect the staggered entry property (c.f. Theorem 3) to hold, but as in Theorem 9, we can establish a performance guarantee associated with "sampling" a solution using the distribution from the corresponding continuous relaxation. Indeed, let $Q_{i}$ denote a random variable that represents the time period when product $i \in I$ is introduced, where the probability distribution of $Q_{i}$ is given by the solution to the continuous relaxation, with $\operatorname{Pr}\left(Q_{i}=t\right)=\bar{x}_{i t}, \forall i \in I, t=1, \ldots, T$. Let $f\left(Q_{1}, \ldots, Q_{n}\right)$ denote the (random) profit associated with the random vector $\left(Q_{1}, \ldots, Q_{n}\right)$, i.e.,

$$
f\left(Q_{1}, \ldots, Q_{n}\right):=\sum_{t=1}^{T} \alpha_{t} \sum_{i=1}^{n} r_{i} \sum_{g=1}^{G} \zeta^{g} \times \frac{\sum_{u=1}^{t} \kappa_{i, t-u}^{g} v_{i}^{g} \mathbb{1}_{\left[Q_{i}=u\right]}}{v_{0}^{g}+\sum_{u=1}^{t} \sum_{i=1}^{n} \kappa_{i, t-u}^{g} v_{i}^{g} \mathbb{1}_{\left[Q_{i}=u\right]}},
$$

where $\mathbb{1}_{A}$ is the indicator function for event $A$. Since $\left(Q_{1}, \ldots, Q_{n}\right)$ is a feasible solution for APP, it follows immediately that $f\left(Q_{1}, \ldots, Q_{n}\right) \leq V^{*}$ almost surely. The following theorem - stated without proof — gives a lower bound for $\mathbb{E}\left[f\left(Q_{1}, \ldots, Q_{n}\right)\right]$.

Theorem 11. Let $\rho:=\min _{g=1, \ldots, G} \frac{v_{0}^{g}}{v_{0}^{g}+\max _{j=1, \ldots, n} v_{j}^{g}}$. Then, $\rho V^{*} \leq \rho \bar{V} \leq \mathbb{E}\left[f\left(Q_{1}, \ldots, Q_{n}\right)\right] \leq V^{*}$.

Beyond this analytical result, we note that we tested our heuristics in instances with two customer classes, and found that their performance is similar to what we have described with a single customer class. This suggests that, although the staggered entry property may not hold, the heuristics developed in this paper still yield relatively good solutions.

\section{Conclusions}

We have presented the $A P P$ for a firm that has to decide when to release a set of products throughout a season. Our formulation assumes that the attractiveness and decay pattern of each product are known in advance. This would be the case if the products have already been designed and produced, like basics and fashion-basics in apparel, and their attractiveness has been estimated, for instance, through marketing techniques, such conjoint analysis, regression models or focus groups. Though the assortment decision for these types of products is usually made only a few 
times a year, planners are constantly evaluating different scenarios. Hence, it pays to have efficient methods to solve the $A P P$.

We have presented here four approximation algorithms for the $A P P$ and we have shown analytically as well as numerically that the overall performance is close to optimal. In particular, the EARLYENTRY and RANDOMIZED algorithms perform very well in our experiments, both on a small scale and with data from the field. In contrast, when some items are much more popular than the rest, then the GREEDY algorithm tends to do best, because the relaxation, which guides EARLyEnTRY and RANDOMIZED, provides a solution far off the optimal integer solution. Finally, we also develop a RuLEOFThumb heuristic that does not need any optimization software, performs well and thus could be used by practitioners to plan the product assortment for a given season.

Furthermore, our experiments suggest that the value of the outside option $v_{0}$ is a key driver of the structure of the optimal solution. On the one hand, when $v_{0}$ is very large (i.e., when the firm has a low market share), then it is best to introduce all products early, i.e., following AlLEARLY. On the other hand, when $v_{0}$ is very small, then introducing the products with the highest margins regardless of the decay pattern is optimal. Outside these two extremes - i.e., for intermediate values of $v_{0}$ - the optimal solution has a more interesting structure and that is when our model and heuristics become most valuable.

One of the limitations of our model is that it assumes that all parameters are well-known to the firm. In particular, the attractiveness and decay patterns are taken as given. If the parameters are highly uncertain and there is an opportunity to gain additional information between decision periods, then a dynamic programming (DP) or closed-loop approach might be more suitable. However, DPs are rarely solvable in practice so researchers resort to approximate methods. One of the most common techniques is the certainty-equivalence approximation that replaces the uncertain parameters by their expected values and resolves the deterministic problem in a rolling horizon fashion (see Bertsimas and Popescu 2003). Hence, this method relies on repeatedly solving the open-loop formulation, for which our model and algorithms would come in handy.

Acknowledgments. The authors thank Yossi Aviv, the AE, and the referees for their helpful and insightful comments, which greatly improve the results and presentation in the paper. Victor Martínez-de-Albéniz's research was supported in part by the European Research Council - ref. ERC-2011-StG 283300-REACTOPS and by the Spanish Ministry of Economics and Competitiveness (Ministerio de Economía y Competitividad, formerly Ministerio de Ciencia e Innovación) - ref. ECO2011-29536. Paat Rusmevichientong's research was supported in part by the National Science Foundation through grants CMMI-1158658, CMMI-1158659, and CMMI-1157569.

\section{References}

Abernathy, F. H., J. T. Dunlop, J. H. Hammond, and D. Weil. 1999. A stitch in time: Lean retailing and the transformation of manufacturing-lessons from the apparel and textile industries. Oxford University 
Press, USA.

Ainslie, A., X. Drèze, and F. Zufryden. 2005. Modeling Movie Life Cycles and Market Share. Marketing Science 24 (3): 508-517.

Allon, G., A. Federgruen, and M. Pierson. 2010. Price Competition Under MNL Demand Functions with Random Coefficients. Working paper, Northwestern University, Kellogg School of Management.

Alptekinoglu, A., D. Honhon, and C. Ulu. 2012. Learning Consumer Tastes Through Dynamic Assortments. Operations Research 60 (4): 833-849.

Aydin, G., and E. Porteus. 2008. Joint inventory and pricing decisions for an assortment. Operations Research 56 (5): 1247-1255.

Bell, D. E., R. L. Keeney, and J. D. C. Little. 1975. A Market Share Theorem. Journal of Marketing Research 12 (2): pp. 136-141.

Benson, H. P. 2007. A simplicial branch and bound duality-bounds algorithm for the linear sum-of-ratios problem. European journal of operational research 182 (2): 597-611.

Bernstein, F., A. G. Kök, and L. Xie. 2010. Dynamic assortment customization with limited inventories. Working paper, Duke University.

Bertsimas, D., and I. Popescu. 2003. Revenue management in a dynamic network environment. Transportation Science 37 (3): 257-277.

Bertsimas, D., C. Teo, and R. Vohra. 1999. On dependent randomized rounding algorithms. Operations Research Letters 24:105 -114.

Besbes, O., and D. Sauré. 2010. Product Assortment and Price Competition Under Multinomial Logit Demand. Working paper, Columbia Graduate School of Business.

Bront, J. M., I. Mendez Diaz, and G. Vulcano. 2009. A column generation algorithm for choice-based network revenue management. Operations Research 57 (3): 769-784.

Caro, F., and J. Gallien. 2007. Dynamic assortment with demand learning for seasonal consumer goods. Management Science 53 (2): 276-292.

Caro, F., and V. Martínez-de-Albéniz. 2012. Product and price competition with satiation effects. Management Science 58 (7): 1357-1373.

Druehl, C. T., G. M. Schmidt, and G. C. Souza. 2009. The optimal pace of product updates. European Journal of Operational Research 192 (2): 621 - 633.

Elberse, A., and J. Eliashberg. 2003. Demand and supply dynamics for sequentially releases products in international markets: The case of motion pictures. Marketing Science 22 (3): 329-354.

Farias, V. F., S. Jagabathula, and D. Shah. 2010. A New Approach to Modeling Choice with Limited Data. To appear in Management Science.

Farias, V. F., and R. Madan. 2011. Irrevocable Multi-Armed Bandit Policies. Operations Research 59 (2): 383-399.

H\&M 2007. Annual report. Technical report, H\&M.

Honhon, D., V. Gaur, and S. Seshadri. 2010. Assortment planning and inventory decisions under stockoutbased substitution. Operations Research 58 (5): 1364-1379.

Honhon, D., and A. G. Kök. 2011. Assortment planning with variety-seeking customers. Presented at the 2010 INFORMS Annual Meeting, Austin, TX.

Kalish, S., V. Mahajan, and E. Muller. 1995. Waterfall and sprinkler new-product strategies in competitive global markets. International Journal of Research in Marketing 12 (2): 105-119.

Kök, A. G., M. Fisher, and R. Vaidyanathan. 2008. Assortment planning: Review of literature and industry practice. In Retail Supply Chain Management: Springer.

Kuno, T. 2002. A branch-and-bound algorithm for maximizing the sum of several linear ratios. Journal of Global Optimization 22 (1): 155-174.

Lim, W. S., and C. S. Tang. 2006. Optimal product rollover strategies. European journal of operational research 174 (2): 905-922.

Mahajan, S., and G. J. van Ryzin. 2001. Stocking retail assortments under dynamic consumer substitution. Operations Research 49 (3): 334-351. 
Moskin, J. 2011. The Perfect Menu. Now Change It. The New York Times February 15.

Raghavan, P., and C. Thompson. 1987. Randomized rounding: a technique for provably good algorithms and algorithmic proofs. Combinatorica 7:365-374.

Rusmevichientong, P., Z.-J. M. Shen, and D. B. Shmoys. 2010. Dynamic assortment optimization with a multinomial logit choice model and capacity constraint. Operations Research 58 (6): 1666-1680.

Sauré, D., and A. Zeevi. 2008. Optimal dynamic assortment planning with demand learning. To appear in $M S O M$.

Savin, S., and C. Terwiesch. 2005. Optimal Product Launch Times in a Duopoly: Balancing Life-Cycle Revenues with Product Cost. Operations Research 53 (1): 26-47.

Schaible, S., and J. Shi. 2003. Fractional programming: the sum-of-ratios case. Optimization Methods and Software 18 (2): 219-229.

Smith, S. A., and N. Agrawal. 2000. Management of Multi-item Retail Inventories Systems with Demand Substitution. Operations Research 48:50-64.

Talluri, K., and G. J. van Ryzin. 2004. Revenue management under a general discrete choice model of consumer behavior. Management Science 50 (1): 15-33.

The Economist 2007. Endless summer. How the business of making blockbusters has changed. The Economist April 26:online.

Tiplady, R. 2006. Zara: Taking the lead in fast-fashion. Business Week April 4.

van Ryzin, G., and S. Mahajan. 1999. On the Relationship Between Inventory Costs and Variety Benefits in Retail Assortments. Management Science 45:1496-1509.

\section{Appendix: Proofs}

\section{Proof of Theorem 1}

Proof. Let $v_{0}$ be given. We show that 2-Period Equal-Margin Assortment Packing is in NP, because we can transform an arbitrary instance of PARTITION, which is a well-known NPcomplete problem, to an equivalent 2-Period Equal-Margin Assortment PaCking problem.

The PARTition problem is defined as follows.

\section{PARTition}

InPUTS: The set of items indexed by $1, \ldots, n$ and the size $c_{i} \in \mathbb{Z}_{+}$associated with each item.

Question: Is there a subset $S \subseteq\{1, \ldots, n\}$ such $\sum_{i \in S} c_{i}=\sum_{i \notin S} c_{i}$ ?

Let $C=\frac{1}{2} \sum_{i=1}^{n} c_{i} \in \mathbb{Z}_{+}$. Since $\sum_{i \in S} c_{i}=\sum_{i \notin S} c_{i}$ if and only if $\sum_{i \in S} c_{i}=C$, we may assume without loss of generality that $C \in \mathbb{Z}_{+}$. An instance of PARTITION can be solved by solving the following instance of 2-PERIOD Equal-MArgin Assortment Packing: define the preference weights as $v_{i}=c_{i}$ for $i=1,2, \ldots, n$, and set the target profit as $K=2 C /\left(v_{0}+C\right)$. The PARTITION problem indeed has a solution if and only if there exists a partition $X_{1}$ and $X_{2}$ such that $\frac{\sum_{j \in X_{1}} v_{j}}{v_{0}+\sum_{j \in X_{1}} v_{j}}+\frac{\sum_{j \in X_{2}} v_{j}}{v_{0}+\sum_{j \in X_{2}} v_{j}} \leq \max _{y \in[0,2 C]}\left\{\frac{y}{v_{0}+y}+\frac{2 C-y}{v_{0}+2 C-y}\right\}=\frac{2 C}{v_{0}+C}=K$. This is true because $G(y)=\frac{y}{v_{0}+y}+\frac{2 C-y}{v_{0}+2 C-y}$ is concave in $y$ over the interval $[0,2 C]$ and achieves a unique maximum at $y=C$. 


\section{Proof of Theorem 2}

Proof. This proof makes use of a later result, Theorem 9, which is proved independently of this theorem. Fix an arbitrary $k$. Clearly the relaxations of $A P P_{k}$ and $A P P$ are identical: $\overline{V_{k}}=\bar{V}$. Given the optimal solution $\left(\bar{\nu}_{i t q}: i=1, \ldots, n, t=1, \ldots, T, q=1, \ldots, k\right)$ associated with the continuous relaxation of $A P P_{k}$, let $\left(Q_{i t q}: i=1, \ldots, n, t=1, \ldots, T, q=1, \ldots, k\right)$ denote the randomized rounding solution as defined by Theorem 9 . It follows that

$$
\left(\frac{v_{0}}{v_{0}+\max _{j=1, \ldots, n} v_{j} / k}\right) \overline{V_{k}} \leq \mathbb{E}\left[f\left(Q_{i t q}: i=1, \ldots, n, t=1, \ldots, T, q=1, \ldots, k\right)\right] \leq V_{k}
$$

where the first inequality follows from the fact that in $A P P_{k}$, the weight of each product $i$ is subdivided into $k$ equal parts and each subproduct has a weight of $v_{i} / k$. Then, we have the following series of inequalities:

$$
\bar{V}=\overline{V_{k}} \geq V_{k} \geq\left(\frac{v_{0}}{v_{0}+\max _{j=1, \ldots, n} v_{j} / k}\right) \overline{V_{k}}=\left(\frac{v_{0}}{v_{0}+\max _{j=1, \ldots, n} v_{j} / k}\right) \bar{V},
$$

which gives the desired result.

\section{Proof of Theorem 3}

Proof. Without loss of generality, we can use in this proof $v_{\ell}=1$ for all $\ell$ (otherwise, we can redefine $\lambda_{\ell}$ below as $\lambda_{\ell} / v_{\ell}$ ). To avoid confusion with the subindices, in this proof we write $x_{i t}$ as $x_{i, t}$. Recalling that $z_{i, t}=\sum_{s=1}^{t} \kappa_{i}^{t-s} x_{i, s}$, we have $z_{i, t}=x_{i, t}+\kappa_{i} z_{i, t-1}$ and $z_{i, 0}=0$. As $J$ denotes the objective function of the continuous relaxation, with preference weights equal to one, we have

$$
J_{i, t}:=\frac{\partial J}{\partial x_{i, t}}=\sum_{l=t}^{T} \kappa_{i}^{l-t} D_{l}=D_{t}+\kappa_{i} J_{i, t+1} .
$$

We know from the KKT conditions that there exists nonnegative dual variables $\lambda_{1}, \ldots, \lambda_{n}$ such that $x_{i, t} \geq 0$ when $J_{i, t}=\lambda_{i}$ and $x_{i, t}=0$ when $J_{i, t}<\lambda_{i}$. We will hence prove that for any $\lambda_{1}, \ldots, \lambda_{n}$, the optimal solution to the relaxed problem has the desired property.

Claim 1: If all $n$ products are introduced, then we can restrict our attention to the case where $\lambda_{1}>\ldots>\lambda_{n}$ and $\lambda_{1}\left(1-\kappa_{1}\right)<\ldots<\lambda_{n}\left(1-\kappa_{n}\right)$.

Proof of Claim 1: Indeed, suppose that $\lambda_{i} \leq \lambda_{i+1}$ for some $i$. Then it is easy to see that for all $i$ and all values of $D_{1}, \ldots, D_{T}>0 J_{i, t}>J_{i+1, t}$ for $t<T$. Since $J_{i, t} \leq \lambda_{i}$, this implies that $J_{i+1, t}<\lambda_{i+1}$ and hence $x_{i+1, t}=0$, for all $t$, i.e., product $i+1$ will never be introduced. Hence $\lambda_{1}>\ldots>\lambda_{n}$.

Similarly, suppose that $\lambda_{i}\left(1-\kappa_{i}\right) \geq \lambda_{i+1}\left(1-\kappa_{i+1}\right)$ for some $i$. Then we prove by induction that for all $t, \lambda_{i}-J_{i, t}>\lambda_{i+1}-J_{i+1, t}$. This is indeed true for $t=T$ because $\lambda_{i}>\lambda_{i+1}$ and $J_{i, T}=J_{i+1, T}=D_{T}$. If the result is true for $t+1$, then it must also be true for $t$ because 
$J_{i, t}=D_{t}+\kappa_{i} J_{i, t+1}$, which implies that $\lambda_{i}-J_{i, t}=\lambda_{i}\left(1-\kappa_{i}\right)-D_{t}+\kappa_{i}\left(\lambda_{i}-J_{i, t+1}\right)>\lambda_{i+1}(1-$ $\left.\kappa_{i+1}\right)-D_{t}+\kappa_{i+1}\left(\lambda_{i+1}-J_{i+1, t+1}\right)=\lambda_{i+1}-J_{i+1, t}$, where the inequality follows from the induction hypothesis. This completes the induction and thus, $\lambda_{i}-J_{i, t}>0$ for all $t$, and product $i$ will never be introduced.

Claim 2: If $x_{i, t}>0$ for some $i$ and $t$, then $D_{t-1} \leq \lambda_{i}\left(1-\kappa_{i}\right) \leq D_{t}$.

Proof of Claim 2: Since $J_{i, t}=D_{t}+\kappa_{i} J_{i, t+1}, x_{i, t}>0$ implies that $J_{i, t}=\lambda_{i}$ and hence $D_{t}=$ $\lambda_{i}-\kappa_{i} J_{i, t+1} \geq \lambda_{i}\left(1-\kappa_{i}\right)$ and $D_{t-1}=J_{i, t-1}-\kappa_{i} \lambda_{i} \leq \lambda_{i}\left(1-\kappa_{i}\right)$.

Claim 3: If $D_{t}>D_{t+1}$ for some $t$, then $D_{t}>D_{t+1}>\cdots D_{T}$ and no product is introduced after period $t$.

Proof of Claim 3: It suffices to show that $D_{t+1}>D_{t+2}$ because the proof for other cases is similar. Suppose on the contrary that $D_{t+2} \geq D_{t+1}$. Then, we have that $D_{t}>D_{t+1}$ and $D_{t+2} \geq D_{t+1}$, which implies that $D_{t+1}^{2}<D_{t} D_{t+2}$, or equivalently,

$$
\left(\frac{\alpha^{t}}{\left(v_{0}+\sum_{i=1}^{n} z_{i, t+1}\right)^{2}}\right)^{2}<\frac{\alpha^{t-1}}{\left(v_{0}+\sum_{i=1}^{n} z_{i, t}\right)^{2}} \cdot \frac{\alpha^{t+1}}{\left(v_{0}+\sum_{i=1}^{n} z_{i, t+2}\right)^{2}} \leq \frac{\alpha^{t-1}}{\left(v_{0}+\sum_{i=1}^{n} z_{i, t}\right)^{2}} \cdot \frac{\alpha^{t+1}}{\left(v_{0}+\sum_{i=1}^{n} \kappa_{i} z_{i, t+1}\right)^{2}},
$$

where the last inequality follows from the fact that $z_{i, t+2}=x_{i, t+2}+\kappa_{i} z_{i, t+1} \geq \kappa_{i} z_{i, t+1}$ for all $i$. Since $D_{t}>D_{t+1}$, it follows that $x_{i, t+1}=0$ for all $i$ by Claim 2. Thus, $z_{i, t+1}=\kappa_{i} z_{i, t}$ for all $i$, and the above inequality implies that $\left(\frac{\alpha^{t}}{\left(v_{0}+\sum_{i=1}^{n} \kappa_{i} z_{i, t}\right)^{2}}\right)^{2}<\frac{\alpha^{t-1}}{\left(v_{0}+\sum_{i=1}^{n} z_{i, t}\right)^{2}} \frac{\alpha^{t+1}}{\left(v_{0}+\sum_{i=1}^{n} \kappa_{i}^{2} z_{i, t}\right)^{2}}$, which implies that $\left(v_{0}+\sum_{i=1}^{n} \kappa_{i} z_{i, t}\right)^{2}>\left(v_{0}+\sum_{i=1}^{n} z_{i, t}\right)\left(v_{0}+\sum_{i=1}^{n} \kappa_{i}^{2} z_{i, t}\right)$ or in other words $\left(\sum_{i=1}^{n} \kappa_{i} z_{i, t}\right)^{2}>$ $\left(\sum_{i=1}^{n} z_{i, t}\right)\left(\sum_{i=1}^{n} \kappa_{i}^{2} z_{i, t}\right)+v_{0}\left(\sum_{i=1}^{n}\left(1-\kappa_{i}\right)^{2} z_{i, t}\right)>\left(\sum_{i=1}^{n} z_{i, t}\right)\left(\sum_{i=1}^{n} \kappa_{i}^{2} z_{i, t}\right)$.

However, an application of Cauchy-Schwarz inequality to vectors $\left(\sqrt{z_{1, t}}, \ldots, \sqrt{z_{n, t}}\right)$ and $\left(\kappa_{1} \sqrt{z_{1, t}}\right.$, $\ldots, \kappa_{n} \sqrt{z_{n, t}}$ ) implies the opposite, which is a contradiction. Thus, $D_{t}$ is strictly decreasing after $t$. Moreover, it follows immediately that no product is introduced after period $t$.

To complete the proof of Theorem 3, we consider the periods when product $i$ is introduced. Define $E_{i}$ the latest period in which $J_{i, E_{i}}=\lambda_{i}$. Note that $x_{i, E_{i}} \geq 0$. Since $J_{i, E_{i}}=D_{E_{i}}+\kappa_{i} J_{i, E_{i}+1}$, it follows that $D_{E_{i}}=\lambda_{i}-\kappa_{i} J_{i, E_{i}+1} \geq \lambda_{i}\left(1-\kappa_{i}\right)$. Let $\widetilde{S}_{i}$ be the smallest $t$ earlier than $E_{i}$ such that $D_{t} \geq \lambda_{i}\left(1-\kappa_{i}\right)$, i.e., $\widetilde{S}_{i}=\min \left\{t: t \leq E_{i}\right.$ and $\left.D_{t} \geq \lambda_{i}\left(1-\kappa_{i}\right)\right\}$. By definition, for any $t<\widetilde{S}_{i}$, $D_{t}<\lambda_{i}\left(1-\kappa_{i}\right)$, which implies that $x_{i, t}=0$ by Claim 2. Similarly, for $\widetilde{S}_{i} \leq t<E_{i}, D_{t}=\lambda_{i}\left(1-\kappa_{i}\right)$, and $x_{i, t} \geq 0$. Since $\lambda_{i}\left(1-\kappa_{i}\right)<\lambda_{i+1}\left(1-\kappa_{i+1}\right)$, then we must have that $\widetilde{S}_{i+1} \geq E_{i}$. 
Furthermore, we now prove that we can define $S_{i} \geq \widetilde{S}_{i}$ such that $x_{i, t}>0$ for $S_{i} \leq t<E_{i}$ and $x_{i, t}=0$ for $\widetilde{S}_{i} \leq t<S_{i}$. Indeed, suppose that there is a period $t$ such that $\widetilde{S}_{i}<t<E_{i}$ and $x_{i, t}=0$. Since only product $i$ could be introduced at $t$, we have $x_{j, t}=0$ for all $j=1, \ldots, n$. Since $D_{t-1}=D_{t}=\lambda_{i}\left(1-\kappa_{i}\right) \leq D_{t+1}$, we have that $D_{t}^{2} \leq D_{t-1} D_{t+1}$. Using the fact that $z_{i, t}=\kappa_{i} z_{i, t-1}$ and $z_{i, t+1} \geq \kappa_{i} z_{i, t}=\kappa_{i}^{2} z_{i, t-1}$ for all $i$, we have that $\left(\frac{\alpha^{t-1}}{\left(v_{0}+\sum_{i=1}^{n} \kappa_{i} z_{i, t-1}\right)^{2}}\right)^{2} \leq$ $\frac{\alpha^{t-2}}{\left(v_{0}+\sum_{i=1}^{n} z_{i, t-1}\right)^{2}} \frac{\alpha^{t}}{\left(v_{0}+\sum_{i=1}^{n} \kappa_{i}^{2} z_{i, t-1}\right)^{2}}$ which generates a contradiction again unless $z_{j, t}=0$ for all $j=1, \ldots, n$, using the same argument as in Claim 3 above. As a result, it must be true that product $i$ is not introduced before time period $t$. Hence we can define $S_{i}$ as the first period after $\widetilde{S}_{i}$ and before $E_{i}$ such that $x_{i, t}>0$. Finally, note that $S_{1}=1$ because otherwise $D_{1}=\frac{1}{v_{0}^{2}}>D_{t}$ for all $t \geq 2$, which would mean that no product is introduced at all.

We have thus shown that each product $i$ is introduced during the interval $\left[S_{i}, E_{i}\right]$ with $\bar{x}_{i t}>0$ for $S_{i} \leq t<E_{i}, \bar{x}_{i, E_{i}} \geq 0$, and $\bar{x}_{i t}=0$ for all $t \notin\left[S_{i}, E_{i}\right]$. Moreover, we have shown that $S_{1}=1$, $E_{i} \leq S_{i+1}$ for all $i$. The last part of Theorem 3 follows from Claims 2 and 3 , and the fact that $\bar{x}_{n, E_{n}-1}>0, \bar{x}_{n, E_{n}} \geq 0$, and $\bar{x}_{j, t}=0$ for all $j$ and $t>E_{n}$.

\section{Proof of Proposition 4}

Proof. Fix an arbitrary $\kappa$. We make use of an equivalent continuous relaxation problem given in terms of $\boldsymbol{z}=\left(z_{1}, \ldots, z_{T}\right)$ where $z_{t}=\sum_{u=1}^{t} \kappa^{t-u} x_{u}=x_{t}+\kappa z_{t-1}$, for any $t \geq 1$. Note that there is a one-to-one correspondence between $\boldsymbol{x}$ and $\boldsymbol{z}$. The optimization problem is given by:

$$
\max \left\{H\left(z_{1}, \ldots, z_{T}\right):=\sum_{t=1}^{T} \frac{\alpha_{t} z_{t}}{v_{0}+z_{t}} \mid \sum_{t=1}^{T}\left(z_{t}-\kappa z_{t-1}\right) \leq v_{1} \text { and } z_{t}-\kappa z_{t-1} \geq 0, t=1, \ldots, T\right\}
$$

where $z_{0}=0$. This is in turn equivalent to the optimization problem:

$$
\max \left\{H\left(z_{1}, \ldots, z_{T}\right) \quad \mid \quad z_{T}+(1-\kappa) \sum_{t=1}^{T-1} z_{t} \leq v_{1} \quad \text { and } \quad \kappa z_{t-1}-z_{t} \leq 0 \quad t=1, \ldots, T\right\}
$$

Let $\overline{\boldsymbol{z}}=\left(\bar{z}_{1}, \bar{z}_{2}, \ldots, \bar{z}_{T}\right)$ denote an optimal solution associated with the above optimization problem, and let $\overline{\boldsymbol{x}}=\left(\bar{x}_{1}, \bar{x}_{2}, \ldots, \bar{x}_{T}\right)$ denote the corresponding optimal product schedule.

By associating the dual variable $\lambda \geq 0$ with the constraint $z_{T}+(1-\kappa) \sum_{t=1}^{T-1} z_{t} \leq v_{1}$, and the variable $\eta_{t} \geq 0$ with the constraint $\kappa z_{t-1}-z_{t} \leq 0$, it follows from the KKT condition that $\bar{z}$ 
satisfies the following set of equations:

$$
\begin{aligned}
& 0=\frac{\partial H}{\partial z_{1}}(\overline{\boldsymbol{z}})-\lambda(1-\kappa)+\eta_{1}-\kappa \eta_{2} \quad \Leftrightarrow \quad \lambda(1-\kappa)+\kappa \eta_{2}=\eta_{1}+D_{1} \\
& 0=\frac{\partial H}{\partial z_{T-1}}(\overline{\boldsymbol{z}})-\lambda(1-\kappa)+\eta_{T-1}-\kappa \eta_{T} \quad \Leftrightarrow \quad \lambda(1-\kappa)+\kappa \eta_{T}=\eta_{T-1}+D_{T-1} \\
& 0=\frac{\partial H}{\partial z_{T}}(\bar{z})-\lambda+\eta_{T} \quad \Leftrightarrow \quad \lambda \quad=\eta_{T}+D_{T}
\end{aligned}
$$

and we have the following complementary slackness condition: $\lambda\left(z_{T}+(1-\kappa) \sum_{t=1}^{T-1} \bar{z}_{t}-v_{1}\right)=0$ and $\eta_{t}\left(\bar{z}_{t}-\kappa \bar{z}_{t-1}\right)=0$ for all $t$.

From Theorem 3 , we know that $\bar{x}_{t}>0$ for $t=1, \ldots, E$ and $\bar{x}_{t}=0$ for $t>E$. We claim that $0=\eta_{1}=\eta_{2}=\cdots=\eta_{E}<\eta_{E+1}<\eta_{E+2}<\cdots<\eta_{T}$. Indeed, it follows immediately from the complementary slackness condition that $\eta_{1}=\eta_{2}=\cdots=\eta_{E}=0$. To prove the strict inequalities, we start showing that $0=\eta_{E}<\eta_{E+1}$. Indeed, if this was not true and $\eta_{E+1}=0$, then $\eta_{E+1}+D_{E+1}<$ $\eta_{E}+D_{E}$, because, from Theorem 3 we know that $D_{E}>D_{E+1}>\cdots>D_{T}$. But we know from the KKT condition that $\eta_{E+1}+D_{E+1}=\lambda(1-\kappa)+\kappa \eta_{E+2} \geq \lambda(1-\kappa)=\lambda(1-\kappa)+\eta_{E+1}=\eta_{E}+D_{E}$, which would be a contradiction. We then prove that $\eta_{E+1}<\eta_{E+2}<\cdots<\eta_{T}$. Suppose on the contrary that $\eta_{E+2} \leq \eta_{E+1}$. This means that $\eta_{E+2}+D_{E+2} \leq \eta_{E+1}+D_{E+1}$. By the KKT condition once again, $\lambda(1-\kappa)+\kappa \eta_{E+3}=\eta_{E+2}+D_{E+2} \leq \eta_{E+1}+D_{E+1}=\lambda(1-\kappa)+\kappa \eta_{E+2}$, which implies that $\eta_{E+3} \leq \eta_{E+2}$. Continuing in this fashion, it follows that $\eta_{T} \leq \eta_{T-1} \leq \cdots \leq \eta_{E+2} \leq \eta_{E+1}$. However, the last two equations in the KKT condition imply that $\eta_{T}=\eta_{T-1}+D_{T-1}-D_{T}>\eta_{T-1}$ because $D_{T}<D_{T-1}$. This is again a contradiction. Therefore, it must be the case that $\eta_{E+2}>\eta_{E+1}$. The same argument shows that $\eta_{E+3}>\eta_{E+2}$, and so on.

Thus far, we have shown that for any arbitrary $\kappa$, there is a cut-off period $E(\kappa)$, after which we do not introduce any product. Moreover, the optimal dual variables $\eta_{1}(\kappa), \eta_{2}(\kappa), \ldots, \eta_{T}(\kappa)$ satisfy the following relationship:

$0=\eta_{1}(\kappa)=\eta_{2}(\kappa)=\cdots=\eta_{E(\kappa)}(\kappa) \quad$ and $\quad 0<\eta_{E(\kappa)+1}(\kappa)<\eta_{E(\kappa)+2}(\kappa)<\cdots<\eta_{T}(\kappa)$ and

$$
E(\kappa)= \begin{cases}\min \left\{t: \eta_{t}(\kappa)>0\right\}-1, & \text { if } \eta_{t}(\kappa)>0 \text { for some } t \\ T, & \text { otherwise. }\end{cases}
$$

For each $t=2, \ldots, T$, let $\bar{\kappa}_{t} \in[0,1]$ be defined by:

$$
\bar{\kappa}_{t}=\inf \left\{\kappa: \eta_{t}(\kappa)>0\right\},
$$


and we set $\bar{\kappa}_{1}=1$. Note that $\eta_{1}(\kappa)=0$ for all $\kappa$ because $x_{1}(\kappa)$ is always positive. Since $\eta_{1}(\kappa) \leq \cdots \leq \eta_{T}(\kappa)$, we have

$$
\left\{\kappa: \eta_{2}(\kappa)>0\right\} \subseteq\left\{\kappa: \eta_{3}(\kappa)>0\right\} \subseteq \cdots \subseteq\left\{\kappa: \eta_{T-1}(\kappa)>0\right\} \subseteq\left\{\kappa: \eta_{T}(\kappa)>0\right\}
$$

which implies that $\bar{\kappa}_{T} \leq \bar{\kappa}_{T-1} \leq \cdots \leq \bar{\kappa}_{3} \leq \bar{\kappa}_{2}$. We claim that

$$
E(\kappa)= \begin{cases}T & \text { if } 0 \leq \kappa<\bar{\kappa}_{T}, \\ T-1 & \text { if } \bar{\kappa}_{T} \leq \kappa<\bar{\kappa}_{T-1}, \\ T-2 & \text { if } \bar{\kappa}_{T-1} \leq \kappa<\bar{\kappa}_{T-2}, \\ \vdots & \vdots \\ 2 & \text { if } \bar{\kappa}_{3} \leq \kappa<\bar{\kappa}_{2}, \\ 1 & \text { if } \bar{\kappa}_{2} \leq \kappa \leq 1,\end{cases}
$$

To see this, note that if $\kappa<\bar{\kappa}_{T}$, it we have $\eta_{T}(\kappa)=0$, which implies that $x_{t}(\kappa)>0$ for all $t$, so $E(\kappa)=T$. At $\kappa=\bar{\kappa}_{T}$, we know that $\eta_{T-1}(\kappa)=0<\eta_{T}(\kappa)$, and thus, $x_{T-1}(\kappa)>0=x_{T}(\kappa)$. For any $\kappa \in\left(\bar{\kappa}_{T}, \bar{\kappa}_{T-1}\right)$, it's easy to see that $x_{T-1}(\kappa)>0$, and $x_{T}(\kappa)$ will remain at zero because, as $\kappa$ increases, the product decays more slowly, and thus, it's beneficial to introduce it earlier. Thus, $E(\kappa)=T-1$ for all $\kappa \in\left(\bar{\kappa}_{T}, \bar{\kappa}_{T-1}\right)$. Using exactly the same argument, we can show $E(\kappa)=t$ for all $\kappa \in\left[\bar{\kappa}_{t+1}, \bar{\kappa}_{t}\right)$. This gives the desired result.

\section{Proof of Proposition 5}

Proof. We proceed as in the proof of Proposition 4 to derive the KKT conditions:

$$
\begin{aligned}
& 0=\frac{\partial H}{\partial z_{1}}(\bar{z})-\lambda(1-\kappa)+\eta_{1}-\kappa \eta_{2} \quad \Leftrightarrow \quad \lambda(1-\kappa)+\kappa \eta_{2}=\eta_{1}+\frac{v_{0} \alpha_{1}}{\left(v_{0}+\bar{z}_{1}\right)^{2}} \\
& 0=\frac{\partial H}{\partial z_{T-1}}(\bar{z})-\lambda(1-\kappa)+\eta_{T-1}-\kappa \eta_{T} \quad \Leftrightarrow \quad \lambda(1-\kappa)+\kappa \eta_{T}=\eta_{T-1}+\frac{v_{0} \alpha_{T-1}}{\left(v_{0}+\bar{z}_{T-1}\right)^{2}} \\
& 0=\frac{\partial H}{\partial z_{T}}(\bar{z})-\lambda+\eta_{T-1} \quad \Leftrightarrow \quad \lambda \quad=\eta_{T}+\frac{v_{0} \alpha_{T}}{\left(v_{0}+\bar{z}_{T}\right)^{2}}
\end{aligned}
$$

with the following complementary slackness conditions: $\lambda\left(z_{T}+(1-\kappa) \sum_{t=1}^{T-1} \bar{z}_{t}-v\right)=0$ and $\eta_{t}\left(\bar{z}_{t}-\kappa \bar{z}_{t-1}\right)=0$ for all $t$.

Since we have a single product, we know from Theorem 3 that there exists $E$ such that $\bar{x}_{t}>0$ for $t \leq E$ and $\bar{x}_{t}=0$ for $t>E$. It follows that for any $k \geq 1, \bar{z}_{E+k}=\kappa^{k} \bar{z}_{E}$, which implies that $\bar{z}_{E} \geq \bar{z}_{E+1} \geq \bar{z}_{E+2} \geq \cdots \geq \bar{z}_{T}$. So, it suffices to show that $\bar{z}_{1} \geq \bar{z}_{2} \geq \cdots \geq \bar{z}_{E}$. We will prove the desired result by contradiction. Suppose on the contrary that $\bar{z}_{k-1}<\bar{z}_{k}$ for some $k \in\{2,3, \ldots E\}$. There are two cases to consider: $k<T$ and $k=T$. 
If $k<T$, since $\bar{x}_{k-1}>0$ and $\bar{x}_{k}>0$, we have $\eta_{k-1}=\eta_{k}=0$, which implies that $\lambda(1-\kappa)=$ $\frac{v_{0} \alpha_{k-1}}{\left(v_{0}+\bar{z}_{k-1}\right)^{2}}$ and $\lambda(1-\kappa)+\kappa \eta_{k+1}=\frac{v_{0} \alpha_{k}}{\left(v_{0}+\bar{z}_{k}\right)^{2}}$. Subtracting the first equation from the second yields $0 \leq \kappa \eta_{k+1}=\frac{v_{0} \alpha_{k}}{\left(v_{0}+\bar{z}_{k}\right)^{2}}-\frac{v_{0} \alpha_{k-1}}{\left(v_{0}+\bar{z}_{k-1}\right)^{2}}=v_{0} \alpha_{k-1}\left(\frac{\alpha}{\left(v_{0}+\bar{z}_{k}\right)^{2}}-\frac{1}{\left(v_{0}+\bar{z}_{k-1}\right)^{2}}\right)<0$, where the last inequality follows from the fact that $\bar{z}_{k}>\bar{z}_{k-1}$. This is a contradiction.

If $k=T$, then again $\eta_{T-1}=\eta_{T}=0$. A similar argument as above implies that $0 \leq \kappa \lambda=$ $\frac{v_{0} \alpha_{T}}{\left(v_{0}+\bar{z}_{T}\right)^{2}}-\frac{v_{0} \alpha_{T-1}}{\left(v_{0}+\bar{z}_{T-1}\right)^{2}}=v_{0} \alpha_{T-1}\left(\frac{\alpha}{\left(v_{0}+\bar{z}_{T}\right)^{2}}-\frac{1}{\left(v_{0}+\bar{z}_{T-1}\right)^{2}}\right)<0$, yielding again a contradiction.

\section{Proof of Theorem 6}

Proof. Assuming $\alpha_{t}=1$ for all $t=1, \ldots, T$, the continuous relaxation of the problem can be recast as an equivalent convex minimization problem:

$$
\min \sum_{t=1}^{T} \frac{1}{v_{0}+z_{t}} \text { s.t. } \sum_{t=1}^{T} z_{t}=W, z_{t} \geq 0, \forall t,
$$

where $W=\sum_{i=1}^{n} v_{i}$. The minimum is achieved by setting $\bar{z}_{t}=W / T$.

Denote $V^{h}$ and $z_{1}^{h}, \ldots, z_{T}^{h}$ the value and loads in each period provided by the heuristic. Note that when $\alpha_{t}=1$ and products last for one period only, items are introduced in decreasing order of $v_{i}$. Without loss of generality, we can assume that no periods are such that they contain the same items in the optimal solution and the heuristic solution, because if so, the ratio $V^{*} / V^{h}$ would become $\left(V^{*}+a\right) /\left(V^{h}+a\right) \geq V^{*} / V^{h}$ where $a$ is the profit obtained in the periods where the two solutions coincide.

Since there are $T$ periods with total load of $W=\sum_{i=1}^{n} v_{i}$ (more generally $W$ would be the total load of the items where optimum and heuristic solution do not coincide), there must be at least one with load equal or larger than $W / T$, and at least one with load equal or smaller than $W / T$.

First, we have a lower bound of $V^{*} \leq \bar{V}=T-\frac{v_{0} T^{2}}{v_{0} T+W}=\frac{T W}{v_{0} T+W}$.

Second, we show that $z_{t}^{h} \in[W /(2 T), 2 W / T]$. If $z_{t}^{h}>2 W / T$ then it is not possible that period $t$ only contains one item, because if so this would also be part of the optimal solution (indeed, because if it had two items in the optimal solution, then it would be possible to increase $V^{*}$ by moving one item to another bin with load smaller than $W / T$ ), contradicting the fact that no bins coincide in the heuristic and optimal solution. Hence, period $t$ has at least two items. Since there is at least a bin with $z_{t^{\prime}}^{h} \leq W / T$, then one should not have added the last item to that bin because there was a bin less full. Hence $z_{t}^{h} \geq 2 W / T$. If $z_{t}^{h}<W /(2 T)$ then we can examine all the bins such that $z_{t}^{h} \geq W / T$ (there is at least one like that). If none of them has more than one item, then again it must be true that all those are part of the optimal solution, which contradicts our 
initial assumption. We can then find one with at least two items. Select the smallest item there, $v_{i} \leq W /(2 T)$. This item was introduced into the bin when the size of the bin was larger than $W /(2 T)$, which contradicts the way the heuristic inserts items into periods.

As a result, we have that

$$
V^{h} \geq T-\max _{z_{t}}\left\{\sum_{t=1}^{T} \frac{v_{0}}{v_{0}+z_{t}} \mid \frac{W}{2 T} \leq z_{t} \leq \frac{2 W}{T} \text { and } \sum_{t=1}^{T} z_{t}=W\right\} .
$$

Since the objective is convex, the maximum is achieved by setting some $z_{t}=\frac{W}{2 T}$ and some others to $z_{t}=\frac{2 W}{T}$ and the last one to another value. Letting $Z_{t}=\left(2 T z_{t} / W-1\right) / 3$, we can transform the maximization above into

$$
\begin{aligned}
& \max \left\{\frac{2 T}{3 W} \sum_{t=1}^{T} \frac{v_{0}}{\frac{2 T v_{0} / W+1}{3}+Z_{t}} \mid 0 \leq Z_{t} \leq 1 \text { and } \sum_{t=1}^{T} Z_{t}=T / 3\right\} \\
& \leq \frac{T v_{0}}{3 W}\left(\frac{\frac{T}{3}}{\frac{2 T v_{0} / W+1}{3}+1}+\frac{\frac{2 T}{3}}{\frac{2 T v_{0} / W+1}{3}}\right)=\frac{T^{2} v_{0}\left(2 T v_{0}+3 W\right)}{\left(T v_{0}+2 W\right)\left(2 T v_{0}+W\right)}
\end{aligned}
$$

Hence,

$$
1 \leq \frac{V^{*}}{V^{h}} \leq \frac{\frac{T W}{T v_{0}+W}}{T-\frac{T^{2} v_{0}\left(2 T v_{0}+3 W\right)}{\left(T v_{0}+2 W\right)\left(2 T v_{0}+W\right)}}=\frac{\left(\frac{T v_{0}}{W}+2\right)\left(\frac{T v_{0}}{W}+\frac{1}{2}\right)}{\left(\frac{T v_{0}}{W}+1\right)^{2}} \leq \frac{9}{8}
$$

because the function $\phi(z)=\frac{(z+2)(z+1 / 2)}{(z+1)^{2}}=1+\frac{z}{2(z+1)^{2}}$ is first increasing until $z=1$ and then decreasing.

\section{Proof of Theorem 8}

Proof. Let $\bar{z}_{i t}=\bar{x}_{i t}+\kappa_{i} \bar{z}_{i t-1}$ and $z_{i t}^{h}=x_{i t}^{h}+\kappa_{i} z_{i t-1}^{h}$ where $\bar{x}_{i t}$ and $x_{i t}^{h}$ are the optimal solution to the relaxation and the EARLyEnTRY decision respectively. From Theorem 3, we know that for each product $i$ there exists $S_{i}, E_{i}$ where the product is introduced during the interval $\left[S_{i}, E_{i}\right]$. The heuristic sets $x_{i t}^{h}=1$ for $t=S_{i}$ and zero otherwise. Hence, for $t<S_{i}, z_{i t}-z_{i t}^{h}=0$; for $S_{i} \leq t \leq E_{i}, \bar{z}_{i t}-z_{i t}^{h} \leq 1-\kappa_{i}^{t-S_{i}}$ : for $t>E_{i}, \bar{z}_{i t}-z_{i t}^{h} \leq\left(1-\kappa_{i}^{E_{i}-S_{i}}\right) \kappa_{i}^{t-E_{i}}$. As a result, $\frac{\bar{z}_{i t}-z_{i t}^{h}}{z_{i t^{h}}} \leq 1_{t>S_{i}}\left(\frac{1}{\kappa_{i}^{E_{i}-S_{i}}}-1\right)$ and hence

$0 \leq \bar{V}-V^{h}=\sum_{t=1}^{T}\left(\frac{\alpha_{t} \sum_{i=1}^{n} v_{i} z_{i t}^{h}}{v_{0}+\sum_{i=1}^{n} v_{i} z_{i t}^{h}}\right)\left(\frac{v_{0}}{v_{0}+\sum_{i=1}^{n} v_{i} z_{i t}} \frac{\sum_{i=1}^{n} v_{i}\left(z_{i t}-z_{i t}^{h}\right)}{\sum_{i=1}^{n} v_{i} z_{i t}^{h}}\right) \leq V^{h} \max _{i=1, \ldots, n}\left\{\frac{1}{\kappa_{i}^{E_{i}-S_{i}}}-1\right\}$.

which leads to the final result.

\section{Proof of Theorem 9}


Proof. We only need to show that $\mathbb{E}\left[f\left(Q_{1}, \ldots, Q_{n}\right)\right] \geq \rho \bar{V}$. For this purpose, note that

$$
\begin{aligned}
\mathbb{E}\left[f\left(Q_{1}, \ldots, Q_{n}\right)\right] & =\sum_{t=1}^{T} \alpha_{t} \sum_{i=1}^{n} r_{i} \mathbb{E}\left[\frac{\sum_{u=1}^{t} \kappa_{i, t-u} v_{i} \mathbb{1}_{\left[Q_{i}=u\right]}}{v_{0}+v_{i} \sum_{u=1}^{t} \kappa_{i, t-u} \mathbb{1}_{\left[Q_{i}=u\right]}+\sum_{j \neq i} v_{j} \sum_{u=1}^{t} \kappa_{j, t-u} \mathbb{1}_{\left[Q_{j}=u\right]}}\right] \\
& \geq \sum_{t=1}^{T} \alpha_{t} \sum_{i=1}^{n} r_{i} \mathbb{E}_{Q_{i}}\left[\frac{\sum_{u=1}^{t} \kappa_{i, t-u} v_{i} \mathbb{1}_{\left[Q_{i}=u\right]}}{v_{0}+v_{i} \sum_{u=1}^{t} \kappa_{i, t-u} \mathbb{1}_{\left[Q_{i}=u\right]}+\sum_{j \neq i} v_{j} \sum_{u=1}^{t} \kappa_{j, t-u} \mathbb{E}_{Q_{j}}\left[\mathbb{1}_{\left[Q_{j}=u\right]}\right]}\right] \\
& =\sum_{t=1}^{T} \alpha_{t} \sum_{i=1}^{n} r_{i} \mathbb{E}_{Q_{i}}\left[\frac{\sum_{u=1}^{t} \kappa_{i, t-u} v_{i} \mathbb{1}_{\left[Q_{i}=u\right]}}{v_{0}+v_{i} \sum_{u=1}^{t} \kappa_{i, t-u} \mathbb{1}_{\left[Q_{i}=u\right]}+\sum_{j \neq i} v_{j} \sum_{u=1}^{t} \kappa_{j, t-u} \bar{x}_{j u}}\right] \\
& \geq \sum_{t=1}^{T} \alpha_{t} \sum_{i=1}^{n} r_{i} \mathbb{E}_{Q_{i}}\left[\frac{\sum_{u=1}^{t} \kappa_{i, t-u} v_{i} \mathbb{1}_{\left[Q_{i}=u\right]}}{v_{0}+v_{i}+\sum_{j \neq i} v_{j} \sum_{u=1}^{t} \kappa_{j, t-u} \bar{x}_{j u}}\right] .
\end{aligned}
$$

The first inequality follows from the fact that the random variables $Q_{j}$ 's are independent, and from applying Jensen's Inequality where we use the fact that the function $x \rightarrow \frac{a}{b+x}$ is convex in $x$. The second equality follows from the fact that $\mathbb{E}_{Q_{j}}\left[1_{\left[Q_{j}=u\right]}\right]=\operatorname{Pr}\left(Q_{j}=u\right)=\bar{x}_{j u}$, and the last inequality follows because $\sum_{u=1}^{t} \kappa_{i, t-u} \mathbb{1}_{\left[Q_{i}=u\right]} \leq 1$ almost surely.

To complete the proof, note that for each product $i$, we have

$$
\begin{aligned}
\mathbb{E}_{Q_{i}}\left[\frac{\sum_{u=1}^{t} \kappa_{i, t-u} v_{i} \mathbb{1}_{\left[Q_{i}=u\right]}}{v_{0}+v_{i}+\sum_{j \neq i} v_{j} \sum_{u=1}^{t} \kappa_{j, t-u} \bar{x}_{j u}}\right] & =\frac{\sum_{s=1}^{t} \bar{x}_{i s} \kappa_{i, t-s} v_{i}}{v_{0}\left(1+\frac{v_{i}}{v_{0}}\right)+\sum_{j \neq i} v_{j} \sum_{u=1}^{t} \kappa_{j, t-u} \bar{x}_{j u}} \\
& \geq\left(\frac{v_{0}}{v_{0}+v_{i}}\right)\left(\frac{\sum_{s=1}^{t} \kappa_{i, t-s} v_{i} \bar{x}_{i s}}{v_{0}+\sum_{j=1}^{n} \sum_{s=1}^{t} \kappa_{j, t-s} v_{j} \bar{x}_{j s}}\right) \\
& \geq\left(\frac{v_{0}}{v_{0}+\max _{j=1, \ldots, n} v_{j}}\right)\left(\frac{\sum_{s=1}^{t} \kappa_{i, t-s} v_{i} \bar{x}_{i s}}{v_{0}+\sum_{j=1}^{n} \sum_{s=1}^{t} \kappa_{j, t-s} v_{j} \bar{x}_{j s}}\right)
\end{aligned}
$$

which implies the desired result.

\section{Appendix: Solving the KKT Conditions For a Single Product Type}

If there is a unique product type with a decay $\kappa$, then there exists $E$ such that in the optimal relaxed solution products are introduced in periods $1, \ldots, E$. Moreover, the market share in each period is $\bar{z}_{t} /\left(v_{0}+\bar{z}_{t}\right)$ such that $\bar{z}_{1} \geq \ldots \geq \bar{z}_{E}$ and these values maximize $\sum_{t=1}^{T} \frac{\alpha_{t} z_{t}}{v_{0}+z_{t}}$, with $z_{t+1}=\kappa z_{t}$ for $t \geq E$ and $\sum_{t=1}^{E}\left(z_{t}-\kappa z_{t-1}\right)=v_{a l l}:=\sum_{i} v_{i}$. The KKT conditions in this case are given by the equations:

$$
\begin{gathered}
\frac{\alpha_{t} v_{0}}{\left(v_{0}+z_{t}\right)^{2}}=\lambda(1-\kappa), \quad t=1, \ldots, E-1, \\
\sum_{t=E}^{T} \frac{\alpha_{t} \kappa^{t-E} v_{0}}{\left(v_{0}+\kappa^{t-E} z_{E}\right)^{2}}=\lambda .
\end{gathered}
$$


Using Equation (18) and $v_{\text {all }}=z_{E}+\sum_{t=1}^{E-1} z_{t}(1-\kappa)$ we obtain

$$
z_{E}=v_{\text {all }}+(1-\kappa)(E-1) v_{0}-\sqrt{\frac{1-\kappa}{\lambda}} \sum_{t=1}^{E-1} \sqrt{\alpha_{t} v_{0}} .
$$

Equation (20) gives an expression for $z_{E}$ which can be substituted in Equation (19) to obtain a fixed point equation in $\lambda$ that can be easily solved in a spreadsheet. 